\title{
Combining STING-based neoantigen- targeted vaccine with checkpoint modulators enhances antitumor immunity in murine pancreatic cancer
}

\author{
Heather L. Kinkead, ${ }^{1}$ Alexander Hopkins, ${ }^{1}$ Eric Lutz, ${ }^{1}$ Annie A. Wu, ${ }^{1}$ Mark Yarchoan, ${ }^{1}$ \\ Kayla Cruz, ${ }^{1}$ Skylar Woolman, ${ }^{1}$ Teena Vithayathil, ${ }^{1}$ Laura H. Glickman, ${ }^{2,3}$ Chudi O. Ndubaku, ${ }^{2}$ \\ Sarah M. McWhirter, ${ }^{2}$ Thomas W. Dubensky Jr., ${ }^{2,4}$ Todd D. Armstrong, ${ }^{1}$ Elizabeth M. Jaffee, ${ }^{1}$ \\ and Neeha Zaidi ${ }^{1}$ \\ 'Sidney Kimmel Comprehensive Cancer Center, Skip Viragh Center for Pancreatic Cancer, The Bloomberg Kimmel \\ Institute for Cancer Immunotherapy, Johns Hopkins University School of Medicine, Baltimore, Maryland, USA. ${ }^{2}$ Aduro \\ Biotechnologies Inc., Berkeley, California, USA. ${ }^{3}$ Actym Therapeutics Inc., Berkeley, California, USA. ${ }^{4}$ Tempest Therapeutics, \\ San Francisco, California, USA.
}

Tumor neoantigens arising from somatic mutations in the cancer genome are less likely to be subject to central immune tolerance and are therefore attractive targets for vaccine immunotherapy. We utilized whole-exome sequencing, RNA sequencing (RNASeq), and an in silico immunogenicity prediction algorithm, NetMHC, to generate a neoantigen-targeted vaccine, PancVAX, which was administered together with the STING adjuvant ADU-V16 to mice bearing pancreatic adenocarcinoma (Panc02) cells. PancVAX activated a neoepitope-specific T cell repertoire within the tumor and caused transient tumor regression. When given in combination with two checkpoint modulators, namely anti-PD-1 and agonist OX40 antibodies, PancVAX resulted in enhanced and more durable tumor regression and a survival benefit. The addition of $0 X 40$ to vaccine reduced the coexpression of $\mathrm{T}$ cell exhaustion markers, Lag3 and PD-1, and resulted in rejection of tumors upon contralateral rechallenge, suggesting the induction of $\mathrm{T}$ cell memory. Together, these data provide the framework for testing personalized neoantigen-based combinatorial vaccine strategies in patients with pancreatic and other nonimmunogenic cancers.

Conflict of interest: Under a licensing agreement between Aduro Biotech Inc. and Johns Hopkins University, the University and EMJ are entitled to fees and royalties on sales of certain cancer vaccine products. This arrangement has been reviewed and approved by the Johns Hopkins University in accordance with its conflict of interest policies.

Submitted: June 21, 2018 Accepted: September 4, 2018 Published: October 18, 2018

\section{Reference information:} JCI Insight. 2018;3(20):e122857. https://doi.org/10.1172/jci. insight.122857.

\section{Introduction}

Cancer cells accumulate tens to thousands of somatic mutations, with the highest mutation rates occurring in mutagen-associated cancers, such as melanoma and non-small cell lung cancer, and cancers associated with DNA-mismatch repair gene defects, such as microsatellite instability high colorectal cancers $(1,2)$. A fraction of these mutations are nonsynonymous and result in altered amino acid-coding sequences, causing tumors to express mutated proteins or neoantigens that are not expressed by normal cells. These neoantigens can potentially be loaded onto MHC molecules and be recognized as foreign by $\mathrm{T}$ cells. The $\mathrm{T}$ cell repertoire involved in their recognition is more likely to escape deletion via central tolerance, making neoantigens attractive targets for personalized vaccines (3). Neoantigens can be identified by whole-exome sequencing (WES) and RNA sequencing (RNASeq), and their immunogenicity can be predicted by MHC I-binding algorithms.

Personalized neoantigen-targeted vaccines have been shown to induce neoepitope-specific $\mathrm{T}$ cells that reject melanoma cells expressing these antigens (4-6). Some hypermutated cancers, such as melanoma, may be sensitive to single-agent immune checkpoint inhibitor therapy because of their high neoantigen burden and abundance of tumor-infiltrating lymphocytes (TILs) (1, 7-9). In contrast, pancreatic adenocarcinomas (PDAs) are less immunogenic tumors with a lower number of predicted neoantigens, low immune infiltration (10), and poor response rates to immune checkpoint inhibitor therapy (11). We and others have shown that GVAX, a GM-CSF-secreting allogeneic vaccine, induces intratumoral lymphoid aggregates in PDA patients to render these tumors immunogenic. These aggregates contain IFN- $\gamma$-producing $\mathrm{CD}^{+}$ 
T cells but display evidence of PD-L1 upregulation (12). Combining GVAX and an anti-CTLA-4 antibody improved survival compared with anti-CTLA-4 monotherapy (13). Together, these data suggest that vaccines effectively induce $\mathrm{T}$ cells that can be activated by immune modulators, such as anti-CTLA-4 and anti-PD-1 antibody therapy.

We therefore hypothesize that vaccines targeted to neoantigens will convert "T cell-poor" tumors into tumors rich in neoepitope-specific $\mathrm{T}$ cells. We further posit that checkpoint modulators will be necessary for a robust and durable antitumor response. Here, we show that a neoantigen-targeted vaccine, PancVAX, comprising twelve 20-mer synthetic long peptides (SLPs) and a STING-based adjuvant, ADU-V19, induced vaccine-specific $\mathrm{T}$ cells within the tumor in a murine pancreatic cancer model, Panc02. Furthermore, the addition of anti-PD-1 antibody to inhibit tolerizing signals and an agonist OX40 antibody to improve priming of low avidity $\mathrm{T}$ cells resulted in enhanced tumor regression and improved survival. Our study provides proof of principle that a combinatorial approach using neoantigen-targeted vaccine, anti-PD-1, and agonist OX40 could be effective in the future immunotherapy of T cell-poor tumors.

\section{Results}

Identification and screening of neoantigens. Panc02 tumor cells were subjected to WES and RNASeq to identify expressed nonsynonymous single nucleotide substitutions (see Methods and Figure 1A). A total of 878 nonsynonymous mutations were identified, with 269 of these predicted to bind either $\mathrm{H} 2-\mathrm{K}^{\mathrm{b}}$ or $\mathrm{H} 2-\mathrm{D}^{\mathrm{b}}$ (the MHC I alleles expressed by Panc02) with an affinity of $\leq 1,000 \mathrm{nM}$. Of these mutations, 150 were in expressed genes. Each mutation was validated using Integrative Genomic Viewer, with mutations present in the mouse mm9 dbsnp database removed (14-16). For all mutations, candidate 8 to 11 amino acid-long peptides were analyzed by the MHC I epitope-binding prediction algorithm NetMHC (versions 3.2, 3.4, and pan 2.8) (17).

SLPs of $>15$ amino acids have previously been shown to induce both $\mathrm{CD}^{+}$and $\mathrm{CD} 8^{+} \mathrm{T}$ cell responses. We therefore synthesized 20 amino acid-long SLPs with mutated residues at position 11 for 239 peptides (Supplemental Table 1; supplemental material available online with this article; https://doi.org/10.1172/ jci.insight.122857DS1). To potentially improve presentation by MHC I, the list also included altered peptide ligands (APLs) with an artificial mutation at position 11 in expressed proteins that were predicted to improve binding affinity (18). Peptides with predicted low-affinity MHC I, which are not expected to induce a response, were also included.

To assess in vivo immunogenicity, wild-type mice were vaccinated subcutaneously with pools of 5 peptides on days 0 and 7 (239 peptides screened in total). At day 13, enriched CD ${ }^{+} \mathrm{T}$ cells fractions were isolated and mixed with TAP-deficient T-2 antigen-presenting cells (APCs) expressing H2-K ${ }^{b}$ or H2- ${ }^{b}$ (19) that had been stimulated with each peptide for 2 hours, and IFN- $\gamma$-producing $C D 8^{+} \mathrm{T}$ cells were determined by ELISPOT. $\mathrm{Ova}_{152-171}$ was used as a positive control. To verify the validity of the T2-based assay as well as to identify putative $\mathrm{CD}^{+}$responses missed by MHC I-restricted T2 cells, whole splenocytes were isolated and pulsed with each peptide overnight, and an ELISPOT assay was performed to detect IFN- $\gamma$-producing cells. Thirty-eight of the two hundred thirty-nine (16\%) peptides were found to be immunogenic by IFN- $\gamma$ ELISPOT (Figure 1B and Supplemental Table 2). Interestingly, immunizations that included peptides 218, 222, 230, and 238 triggered IFN- $\gamma$ production only when whole splenocytes were pulsed with peptide but not when enriched $\mathrm{CD}^{+} \mathrm{T}$ cells were stimulated with the T2 APCs (data not shown). This suggested the possible induction of $\mathrm{CD}^{+} \mathrm{T}$ cell reactivity $\left(\mathrm{T} 2-\mathrm{K}^{\mathrm{b}}\right.$ and $\mathrm{T} 2-\mathrm{D}^{\mathrm{b}}$ cells do not express $\mathrm{MHC}$ II and, therefore, cannot stimulate a $\mathrm{CD} 4^{+}$response).

Furthermore, when mice were implanted with Panc02 cells and/or administered a whole tumor cell vaccine comprising irradiated Panc02 cells and the bystander $\mathrm{B} 78 \mathrm{H} 1$ cells that secrete granulocyte-macrophage colony-stimulating factor (GM-CSF), restimulation by a sampling of individual peptides did not yield significant $\mathrm{CD}^{+} \mathrm{T}$ cell responses by ELISPOT. This demonstrated that preexisting $\mathrm{CD} 8^{+} \mathrm{T}$ cells to these epitopes could not be induced with a whole tumor cell vaccine developed from the same tumor that expresses the neoepitopes (Supplemental Figure 1).

Epitope mapping and cross-reactivity to wild-type peptide. Our criteria for developing a neoepitope-targeted vaccine required the inclusion of neoepitopes that are both expressed (as determined by RNASeq) and immunogenic. Because there are no current methods to predict the optimal immunogenic neoepitopes, we expected that both strongly immunogenic and weakly immunogenic epitopes would be included using available prediction approaches. The final Panc02 neoepitope vaccine that was used for further analysis included SLPs for 9 neoantigens and 3 APLs (Supplemental Table 4). 


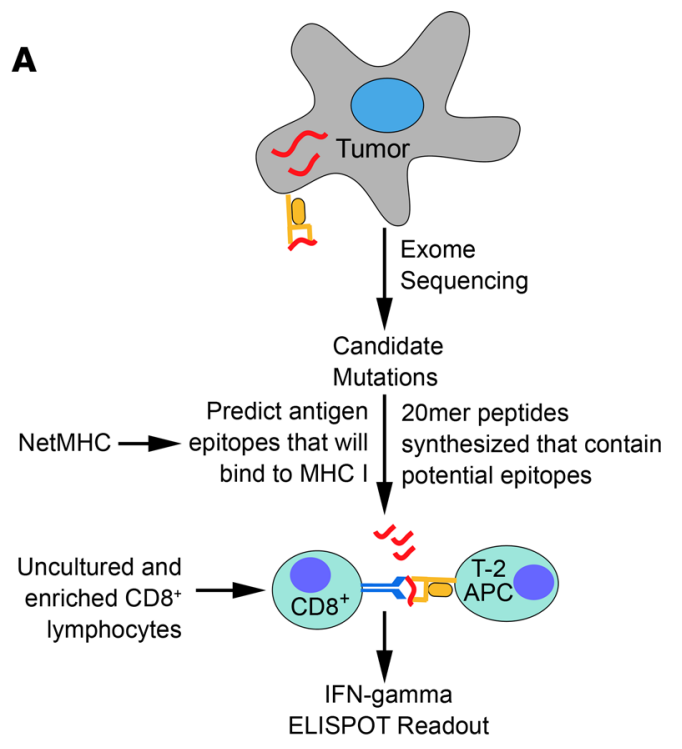

$\mathbf{B}$

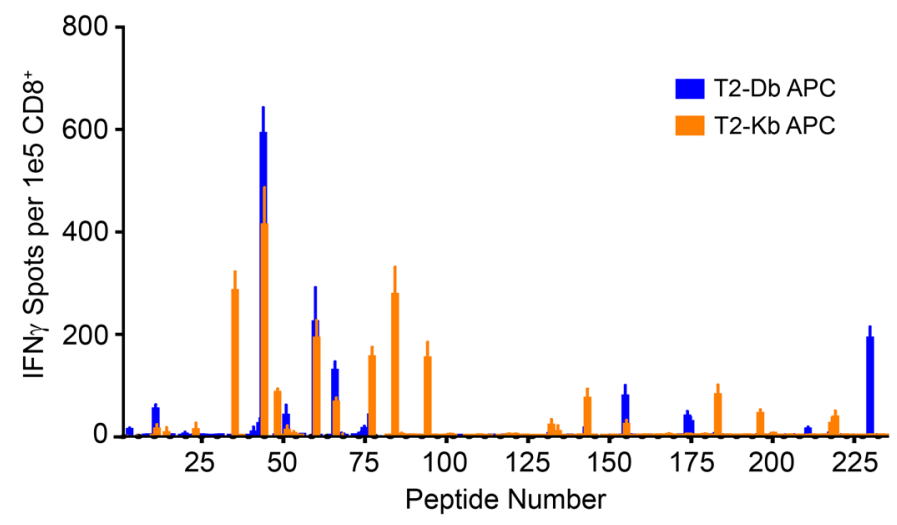

C

D
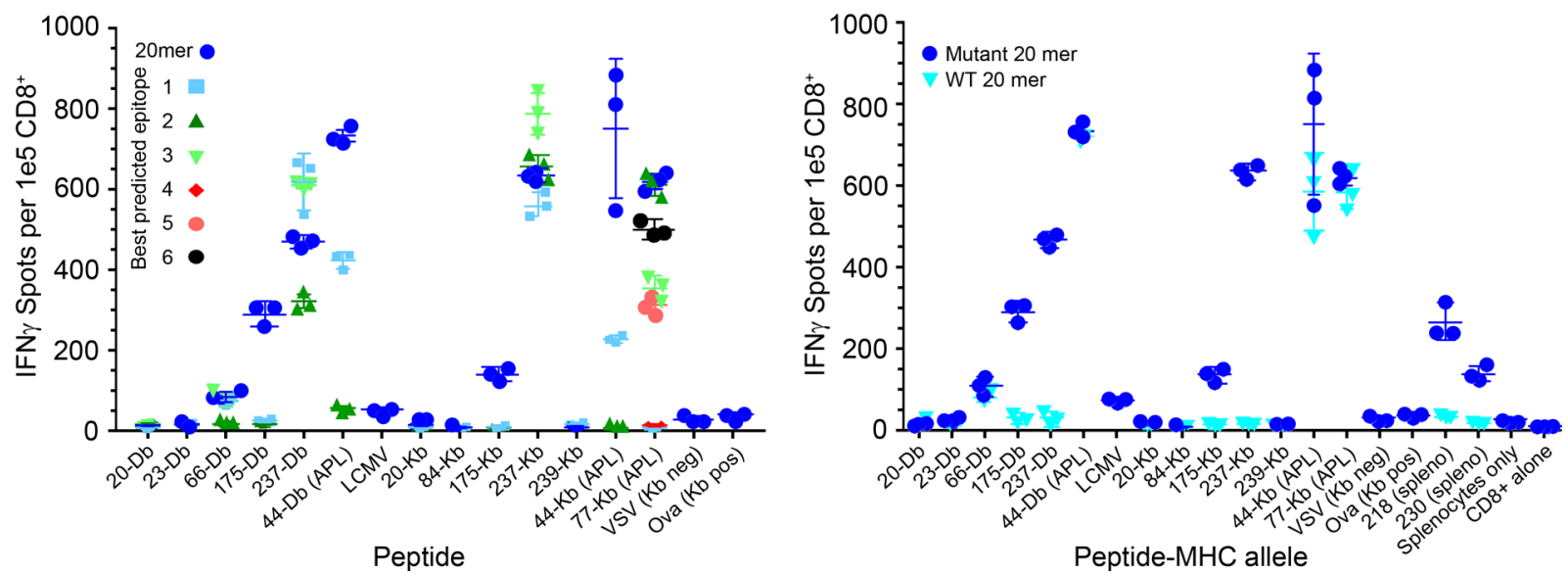

Figure 1. Neoantigen prediction pipeline and estimation of immunogenicity. (A) Tumor neoepitope identification pipeline from whole-exome sequencing (WES) to in vivo verification. (B) C57BL6) mice were immunized with 20-mer peptides corresponding to mutant Panc02 peptides identified in Supplemental Table 4. Isolated CD8 ${ }^{+} \mathrm{T}$ cells were stimulated with $\mathrm{T2}-\mathrm{D}^{\mathrm{b}}$ or $\mathrm{K}^{\mathrm{b}} \mathrm{APC}$ s pulsed with cognate peptides on an IFN- $\gamma$ capture plate and resulting spots were counted (ELISPOT assay, see Methods). (C) Mice were immunized with pooled 20-mer peptides, and isolated CD8 ${ }^{+}$cells were tested for reactivity to minimal epitopes that were predicted from NetMHC algorithm (Supplemental Table 3). Altered peptide ligands (APLs) are indicated in the graph. The peptide number is followed by the MHC-restricted allele ( $\mathrm{D}^{\mathrm{b}}$ or $\mathrm{K}^{\mathrm{b}}$ ) on the $x$ axis. Symbols represent a single mouse together with mean $\pm \mathrm{SEM}$ ( $n=3$ mice per group). Negative controls were VSV and LCMV peptides (see Methods). (D) Mice were immunized with pooled 20-mer peptides corresponding to the mutant neoepitopes and APLs, and isolated CD8 ${ }^{+} T$ cells were analyzed for cross-reactivity to the wild-type 20-mer.

To further characterize these peptides, mapping experiments were performed to determine the minimal epitopes of the respective 20-mers and to test their comparative efficacy in inducing IFN- $\gamma$-producing $\mathrm{CD}^{+} \mathrm{T}$ cells (Figure $1 \mathrm{C}$ ). We synthesized 8 to 11 amino acid-long peptides corresponding to the expected minimal immunogenic epitopes with predicted binding affinities of $\leq 1,000 \mathrm{nM}$ using NetMHC (Supplemental Table 3). To evaluate the immunogenicity of these minimal-length epitopes, mice were immunized with 20-mer SLPs on day 0 and day 7, and splenocytes were isolated on day 13. ELISPOT analysis was performed using either 20-mers or the respective minimal epitopes to determine epitope specificities (Figure 1C). Of note, the immunogenicity of certain 20-mers, such as 44 and 175 , exceeded that of the respective minimal epitope peptides (Figure 1C). In contrast, immunogenicity of the minimal epitope for peptide 237 somewhat exceeded that of the parent 20-mer. It was interesting to observe larger responses using the 20 -mer over the predicted exact binding epitope for many of the peptides. This could be due to the presence of multiple epitopes within the 20-mers and/or alterations in antigen processing that were not possible with 
the exact peptides. Since the 20-mers were largely more effective than the respective minimal epitope peptides, these data supported our rationale for using SLPs as has previously been shown (20).

For the identified immunogenic epitopes, it would be expected that the mutated residue will either be recognized by the $\mathrm{T}$ cell receptor, because it is different from the self-peptide or will improve recognition of the native peptide sequence through improved binding and/or loading onto MHC I. Furthermore, APLs could potentially improve recognition of the native peptide sequence. The altered amino acid of the APL would likely fall in an anchor position when mapped. The presumed mechanism would be that the altered amino acid would create a more optimal anchor residue, thereby increasing the affinity of the peptide for the MHC molecule, which effectively would result in improved priming of $\mathrm{T}$ cells reactive to the native epitope.

To determine the cross-reactivity to the native peptide sequences, mice were immunized with the 12-peptide neoepitope vaccine on days 0 and 7. ELISPOT assays were performed on day 13 using T2-D or $\mathrm{T} 2-\mathrm{K}^{\mathrm{b}}$ APCs pulsed with either the 20-mer native peptide or the corresponding mutant 20-mer peptide (Figure 1D). There was limited cross-reactivity between mutated and native peptides. Two of the three peptides that cross reacted were APLs (peptides 44 and 77) and were able to induce similar levels of IFN- $\gamma$-producing $\mathrm{CD} 8^{+} \mathrm{T}$ cells when restimulated with either wild-type or mutated peptide (Figure 1D). However, only 1 of the 9 neoepitope peptides (peptide 66) tested was also capable of inducing a cross-reactive response, despite response to the mutant peptide (Figure 1D). We also demonstrated lack of cross-reactivity of the neoantigens toward shared tumor antigens, such as mesothelin (Supplemental Figure 2). In this experiment, mice were similarly vaccinated with neoepitope peptides on days 0 and 7 . ELISPOT was performed on day 13, and APCs were restimulated with either the neoepitope or shared antigen (meso-3 or meso-6). This experiment confirms a lack of cross-reactivity to mesothelin, which is known to be expressed in Panc02 tumor cells (21).

A STING pathway-targeted adjuvant enhances vaccine immunogenicity. The antitumor activity of any cancer vaccine requires potent adjuvants that initiate both innate and adaptive immune signals to ultimately induce optimal $\mathrm{T}$ cell activation and persistent function within an immunosuppressive tumor microenvironment (TME). To optimize the $\mathrm{T}$ cell response to our peptide vaccine, we compared the efficacy of 5 adjuvants that have been shown to elicit robust, vaccine-specific, $\mathrm{CD} 8^{+} \mathrm{T}$ cell responses in clinical trials. Polyinosine-polycytidylic acid (poly IC:LC), a double-stranded RNA synthetic analog, is a TLR3 agonist that triggers type I IFN and IL-12 production and promotes Th1/CD8 ${ }^{+} \mathrm{T}$ cell immunity (22-25). ML RR-S2 cGAMP (ADU-V19) is a human STING-activating cyclic dinucleotide (CDN), a derivative of the natural STING ligand produced by cGAS that is modified chemically to be resistant to phosphodiesterase and able to activate all 5 human STING alleles (Aduro Biotechnologies) (Figure 2A). ADU-V19 displays potent antitumor activity by inducing type I IFN production, BATF3 lineage dendritic cell maturation, and T effector cell recruitment and activation (data not shown) (26). Montanide ISA51 and Montanide ISA720 (Seppic Pharmaceuticals) induce $\mathrm{CD}^{+}$and $\mathrm{CD}^{+} \mathrm{T}$ cell responses, respectively $(27,28)$. Finally, AddaVax is a $2 \%$ squalene, with water composition similar to that of the approved adjuvant MF59; it was used to coformulate neoantigen peptides and innate immune agonists to facilitate codelivery of the vaccine components into the same APCs. AddaVax elicits both $\mathrm{CD} 8^{+}$and $\mathrm{CD} 4^{+}$immunity $(29,30)$ and has been used together with the poly IC:LC and STING adjuvants (31).

Mice were immunized with the 12 Panc02 peptides (pooled) and each adjuvant at days 0 and 7 . CD8 ${ }^{+}$ $\mathrm{T}$ cells were enriched from isolated splenocytes on day 13 and then restimulated in vitro with the individual immunizing SLPs for ELISPOT. Peptides 44 and 237 in particular elicited robust responses when ADU-V16 and AddaVax were used as adjuvants (Figure 2B). We further evaluated the effect of the Panc02 peptide vaccine-ADU-V16-AddaVax combination (termed PancVAX) on polycytokine production by $\mathrm{CD} 8^{+} \mathrm{T}$ cells using flow cytometry. Enriched $\mathrm{CD}^{+} \mathrm{T}$ cells from vaccinated mice were incubated overnight with $\mathrm{T} 2 \mathrm{APCs}$ in the presence of protein transport inhibitors and peptides 44 and 237, with ovalbumin as a negative control (Figure 2C). More than $10 \%$ of the isolated CD8 ${ }^{+} \mathrm{T}$ cells expressed IFN and PD- 1 when restimulated with peptides 44 or 237 , and $60 \%-80 \%$ of the IFN- $\gamma^{+} \mathrm{PD}-1^{+} \mathrm{CD} 8^{+} \mathrm{T}$ cell population also expressed TNF- $\alpha$ and granzyme $\mathrm{B}$ (Figure 2C). These data supported the further testing of this 12-peptide vaccine with the ADU-V16 adjuvant and AddaVax (i.e., PancVAX). Of note, we also did not find an increase in immunogenicity to the selected neoepitopes when mice were treated with adjuvant (ADU-V16-AddaVax) alone (Supplemental Figure 3).

PancVAX displays antitumor efficacy in tumor-bearing mice. Three days following the implantation of $10^{6}$ Panc02 cells, mice were vaccinated with the 12 Panc02 peptides (50 $\mu$ g of each peptide shown in Supplemental Table 4) plus AddaVax with either poly IC:LC or ADU-V16 (i.e., PancVAX) (Figure 3A). A 
A

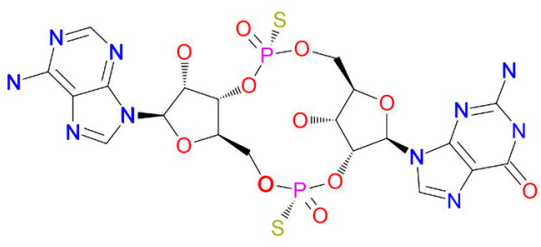

B

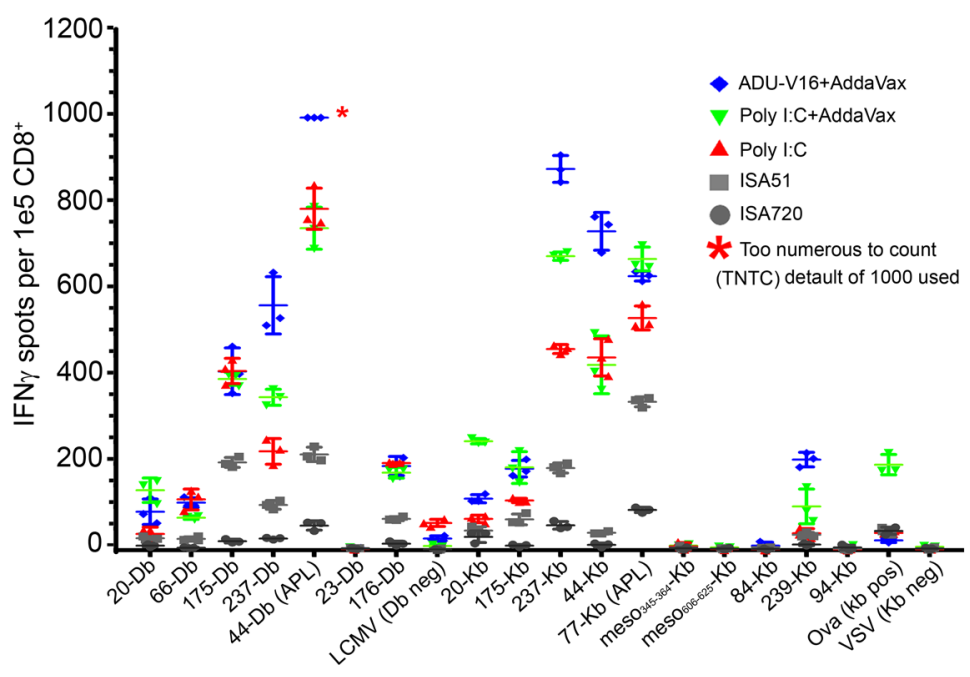

C Peptide 44

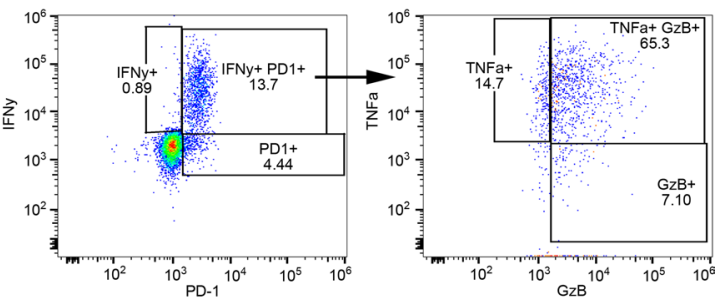

Peptide 237
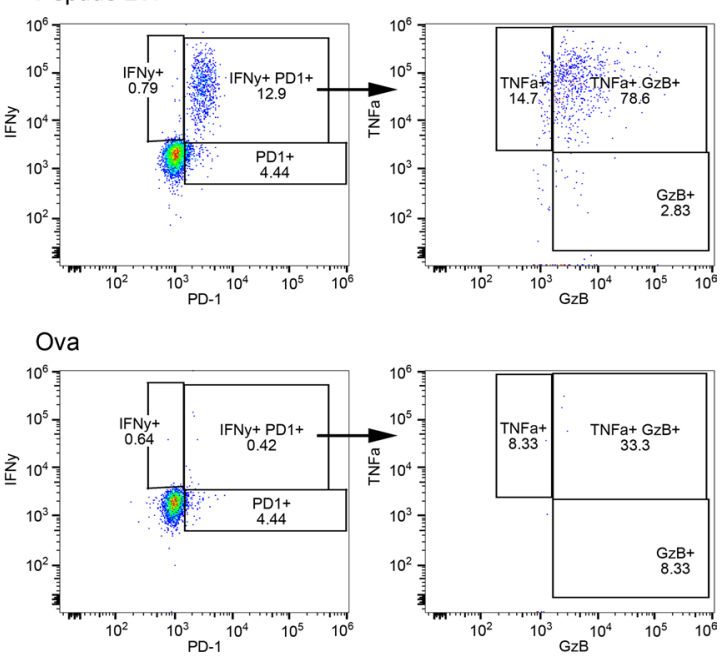

Figure 2. Optimizing adjuvant choice in combination with peptide vaccine. (A) Chemical structure of the STING-based agonist adjuvant ADU-V16 (Aduro Biotech). (B) Naive [57BL6J mice were immunized with pooled 20-mer peptides corresponding to the mutant neoepitopes and altered peptide ligands (APLs), together with adjuvants, namely ADU-V16, R287, poly I:C, ISA51, ISA720, or AddaVax. Isolated CD8 ${ }^{+}$T cells were analyzed for IFN- $\gamma$ production (ELISPOT, see Methods). Wells that were saturated were marked as too numerous to count (TNTC), with a default value of 1,000 spots. APLs are indicated in the graph after the peptide identifier, followed by the MHC-restricted allele $\left(D^{\mathrm{b}}\right.$ or $\left.\mathrm{K}^{\mathrm{b}}\right)$. Symbols represent a single mouse; mean \pm SEM $(n=3$ mice per group). (C) Naive C57BL6] mice were immunized with Panc02 peptides ( $50 \mu \mathrm{g}$ of each if the twelve 20 -mer peptides), ADU-V16, and AddaVax. Overnight stimulation with 2 Panc02 peptides, 44 and 237, presented by T2 APCs resulted in a robust, polyfunctional CD8 ${ }^{+} \mathrm{T}$ cell response (flow cytometry, representative data from single mouse). Live, $\mathrm{CD}^{+} \mathrm{CD}^{+} \mathrm{T}$ cells were gated and graphed for IFN- $\gamma$ and PD-1 expression. The granzyme B and TNF- $\alpha$ graph shows cells gated on both IFN- $\gamma$ and PD-1, as denoted by the arrows. Ovalbumin stimulation was used a negative control.

booster dose was given on day 10, with average tumor diameter measured every 3 to 4 days until tumors reached an average diameter of $10 \mathrm{~mm}$, at which point the mice were euthanized (Figure 3A). Time to measurable tumor (Kaplan-Meier) was delayed significantly in mice treated with PancVAX compared with other groups, notably, the peptide-Poly IC:LC-AddaVax group, which also displayed immunogenicity (Figure 2C and Figure 3B). Tumor diameter measurements indicated a significant suppressive effect of PancVAX at day 20 but with a loss of responsiveness at further time points (Figure 3C).

To test which $\mathrm{T}$ cell subset was mediating the antitumor effect of PancVAX, the experiments were repeated in mice in which $\mathrm{CD} 8^{+}$or $\mathrm{CD} 4^{+} \mathrm{T}$ cells were depleted with anti-CD8 and anti-CD4 $\mathrm{T}$ cell-depleting antibodies, respectively. $\mathrm{CD} 8^{+} \mathrm{T}$ cell depletion completely abrogated the protective effect of PancVAX, while a partial reversal was noted upon $\mathrm{CD}^{+} \mathrm{T}$ cell depletion (Figure $3 \mathrm{D}$ ). In this model, therefore, $\mathrm{CD} 8^{+}$ $\mathrm{T}$ cells appear to be the main drivers of tumor rejection.

As PancVAX alone did not show durable tumor control, we hypothesized that immunosuppressive mechanisms within the TME were responsible for tumor escape. FACS analysis showed that, in PancVAX-treated mice, TILs expressed several checkpoint and exhaustion markers, namely PD-1, Lag3, and Tim3, as well as the transcription factor Tbet (Figure 3E). The latter indicated $\mathrm{T}$ cell exhaustion, which is potentially rescuable by checkpoint blockade (32). In separate experiments, Panc02 cells were cultured in the presence or absence of mouse IFN- $\gamma(10 \mathrm{ng} / \mathrm{ml})$ for 72 hours and labeled using anti-mouse PD-L1 or control isotype antibody. FACS showed that IFN- $\gamma$ enhanced PD-L1 expression on Panc02 tumor cells (Figure $3 \mathrm{~F}$ ). The latter finding recapitulates our previously reported results in mice treated with a whole tumor cell vaccine in a Panc02 metastatic hemispleen model (33). 
A

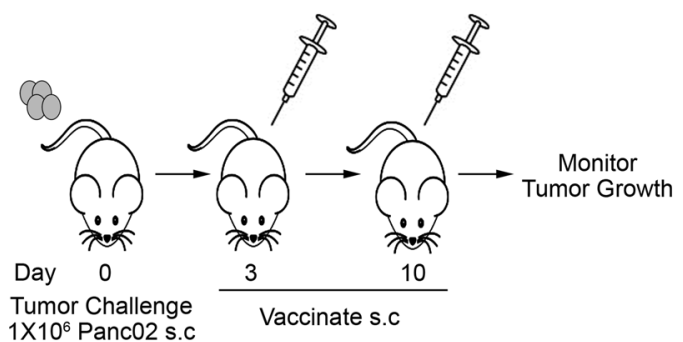

B

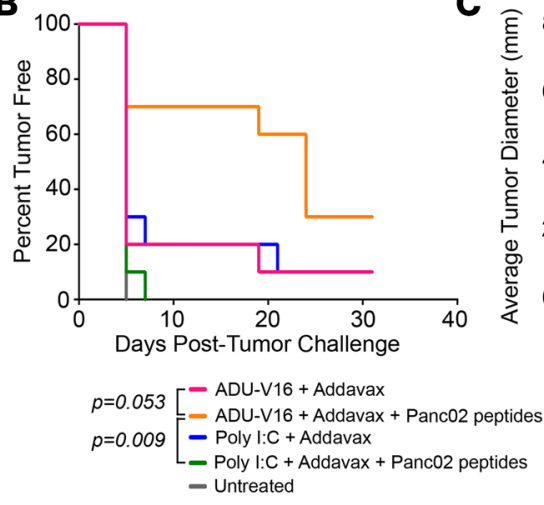

C

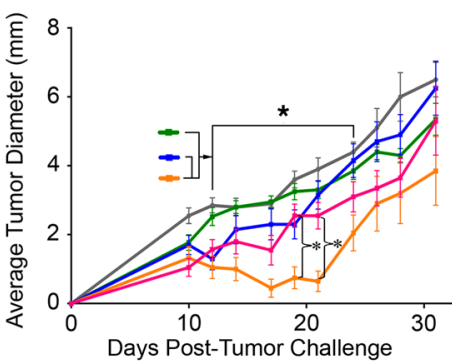

- ADU-V16 + Addavax

- ADU-V16 + Addavax + Panc02 peptides

- Poly I:C + Addavax

- Poly I:C + Addavax + Panc02 peptides

- Untreated
D

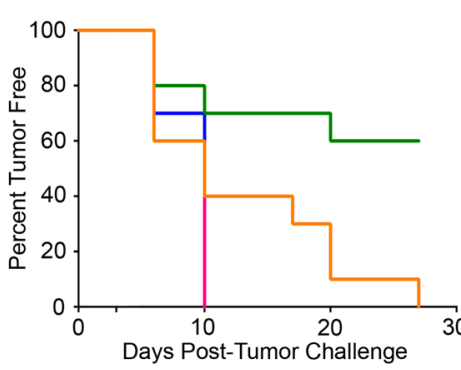

- PancVAX + CD8 Depletion * $\begin{aligned} & \text { * PancVAX + CD4 Depletion } \\ & * \text { - PancVAX + CD4 and CD8 Depletion } \\ & * \text { - PancVAX + Isotype Abs }\end{aligned}$
E

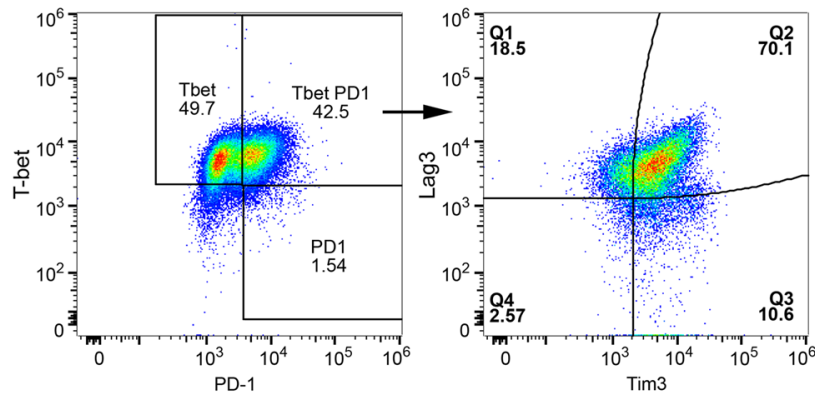

F

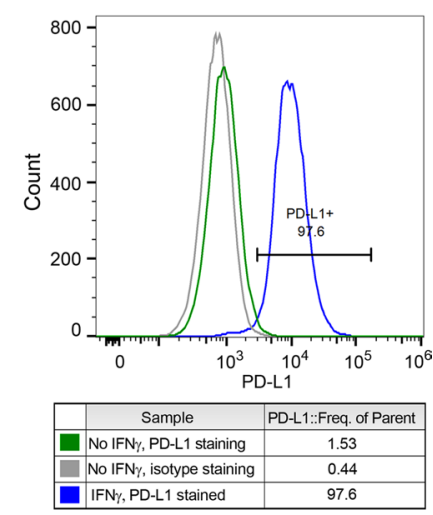

Figure 3. Tumor-specific peptides in combination with ADU-V16 and AddaVax result in tumor regression and a survival benefit. (A) Schematic representation of the vaccination schedule. Mice were challenged with $10^{6}$ Panc02 tumor cells in the right hind leg on day 0 , followed by vaccination, at days 3 and 10, with Panc02 peptides together with AddaVax $(50 \mu \mathrm{g})$ and either Poly I:C (10 $\mu \mathrm{g})$ or ADU-V16 $(5 \mu \mathrm{g})$. Mice were euthanized when the tumors reached 10 $\times 10 \mathrm{~mm}$ or when tumors began to impair mobility or ulcerate. (B) Kaplan-Meier curves showing the percentage of mice that were tumor free. Statistics by log-rank Mantel-Cox test. (C) Tumor growth was measured every 3 to 4 days with calipers until tumors reached $10 \times 10 \mathrm{~mm}$. ${ }^{*} P<0.001$, Student's $t$ test, corrected for multiple comparisons. $n=10$ mice in each treated group, 5 mice in untreated group for both $\mathbf{B}$ and $\mathbf{C}$. (D) Mice were similarly vaccinated as in A and were either depleted of CD4+ or CD8 ${ }^{+} \mathrm{T}$ cells or both with the respective antibodies (see Methods). Kaplan-Meier curves showing the percentage of mice that were tumor free. $n=10$ mice per group at outset. Statistics by log-rank Mantel-Cox test. (E) Flow cytometry for cell surface exhaustion markers of tumor-infiltrating lymphocytes from mice treated with PancVAX, ADU-V16, and AddaVax. Live, CD3 ${ }^{+} C D 8^{+}$Tbet ${ }^{+} P D 1^{+} T$ cells were gated and graphed for Tim3 and Lag3 expression (representative data shown). (F) Panc02 cells were cultured in the presence or absence of $10 \mathrm{ng} / \mathrm{ml} \mathrm{mouse} \mathrm{IFN-} \gamma$ for 72 hours. Cells were stained with anti-mouse PD-L1 or control isotype antibody and analyzed by flow cytometry (shown as representative traces).

Checkpoint modulation enhances the antitumor activity of PancVAX. Given the upregulation of several checkpoints and exhaustion markers following PancVAX, we sought to improve the durability of the antitumor response by modulating two checkpoint molecules, PD-1 and OX40. We have shown that PD-1 blockade augments our whole tumor cell vaccine to eradicate Panc02 liver metastases (33). We also previously reported that OX40 costimulation prolongs the life span of vaccine-activated tumor-specific $\mathrm{T}$ cells in tumor-bearing HER-2/neu-transgenic mice. This strategy prevented exhaustion and converted ineffective low-avidity HER-2/neu-specific $\mathrm{CD} 8^{+} \mathrm{T}$ cells into fully activated $\mathrm{T}$ cells that eradicated HER-2/neu-expressing tumors in tolerant mice (34).

Mice were implanted with Panc02 cells in the hind limb as before, and tumors were allowed to grow until they were palpable at day 7. The mice were then dosed with PancVAX, anti-PD1 antibody (100 $\mu \mathrm{g})$, and agonist OX40 antibody (150 $\mu \mathrm{g})$ (timeline in Figure 4A). Tumor diameters were measured every 3 to 4 days. This "triple treatment" led to near-complete tumor eradication, whereas PancVAX plus single checkpoint blockade gave a significant tumor-free delay compared with PancVAX alone (Figure 4B). For statistical analysis of tumor diameter, data up to 35 days were used, after which the groups became unequal due to loss of mice. Triple-treated mice showed a significant decrease in average tumor diameters compared with mice treated with PancVAX alone (Figure 4C). Figure 4D shows the tumor diameter of each mouse over time. 
As OX40, at a high dose of $150 \mu \mathrm{g}$, itself displayed antitumor activity (Supplemental Figure 3), we sought to isolate the effect of PancVAX from that of OX40 and, importantly, define the minimal effective dose of the OX40 antibody that could synergize with PancVAX and anti-PD-1. Treatments were started 14 days following Panc02 implantation, at which time, tumors were even more well established (Figure 4A, right). Injection of one-third of the OX40 dose, $50 \mu \mathrm{g}$, did not affect tumor size, but when used with PancVAX and anti-PD-1 antibody, significantly reduced tumor diameter (Figure 4E). Triple therapy with low-dose OX40 also significantly improved survival (Figure 4F), with $30 \%$ of mice being tumor free (data not shown). This experiment established that OX40 at a low dose enhances the antitumor effect of PancVAX and anti-PD-1.

Triple therapy induces vaccine-specific TILs and alters T cell phenotype. $\mathrm{CD} 8^{+} \mathrm{T}$ cell fractions were isolated from tumors of triple-treated mice or mice treated with isotype controls. The cells were mixed with TAP-deficient T-2 APCs expressing $\mathrm{H} 2-\mathrm{K}^{\mathrm{b}}$ or $\mathrm{H} 2-\mathrm{D}^{\mathrm{b}}$ that had been stimulated with individual PancVAX peptides for 2 hours, following which IFN- $\gamma$-producing $C D 8^{+} \mathrm{T}$ cells were determined by ELISPOT. Multiple PancVAX peptides elicited robust $\mathrm{CD} 8^{+} \mathrm{T}$ cell IFN- $\gamma$ responses, whereas cells from mice receiving isotype control did not (Figure 5, A and B). This experiment documented the induction and activation by triple therapy of vaccine-specific effector T cells in tumor tissue.

In separate studies, flow cytometry of disaggregated TILs isolated on day 43 from mice treated with either PancVAX plus agonist OX40 antibody or PancVAX plus anti-PD-1 antibody showed differences in IFN- $\gamma$ and PD-1 expression on CD8 ${ }^{+} \mathrm{T}$ cells. Notably, cells isolated from PancVAX-OX40-treated mice showed robust IFN- $\gamma$ production with minimal PD-1 expression. In contrast, mice treated with PancVAX plus antiPD-1 displayed low IFN- $\gamma$ expression and high surface PD-1 (Figure 5C). Moreover, with OX40 treatment, $\mathrm{CD}^{+}{ }^{+} \mathrm{CD} 4{ }^{+} \mathrm{FoxP}^{+}$Tregs were reduced and $\mathrm{IFN}-\gamma$-expressing $\mathrm{CD}^{+}{ }^{+} \mathrm{CD} 4^{+} \mathrm{T}$ cells were increased in the TME. The latter finding indicates that OX40 activation shifts the inducible Treg compartment toward a $\mathrm{T}$ effector phenotype (Figure 5D). Analysis of TILs for exhaustion markers further showed that PancVAX plus anti$\mathrm{PD}-1$ treatment increased the $\mathrm{CD} 8^{+} \mathrm{PD}-1^{+} \mathrm{Lag} 3^{+}$cell population, while addition of the agonist $\mathrm{OX} 40$ antibody resulted in marked decreased expression of exhaustion markers (Figure 5E).

With low-dose OX40 (50 $\mu \mathrm{g} /$ mouse), we similarly found a significant reduction in tumor-infiltrating $\mathrm{CD}^{+} \mathrm{T}$ cells that coexpressed PD-1 and Lag3 (Figure 5F). Importantly, OX40 alone did not reduce $\mathrm{PD}-1^{+} \mathrm{Lag} 3^{+} \mathrm{CD} 8^{+} \mathrm{T}$ cells, suggesting that PancVAX is necessary in addition to checkpoint modulation to effectively reduce $\mathrm{T}$ cell exhaustion. An additional explanation is that OX40 agonist activity only works on newly activated $\mathrm{T}$ cells and cannot reverse an exhaustion phenotype of preexisting $\mathrm{T}$ cells (35).

Triple therapy results in a durable antitumor response. To study the durability of the antitumor response induced by PancVAX, the 3 mouse groups that displayed varying extents of tumor clearance, namely triple therapy, PancVAX plus OX40, and PancVAX plus anti-PD-1, were rechallenged with Panc02 tumors on the contralateral hind limb on day 56 after the initial tumor challenge. No further therapy was initiated, and tumor growth was monitored every 3 to 4 days. Consistent with the initial results, 4 of 5 mice from the triple-treated group showed near-complete tumor eradication, whereas PancVAX-OX 40 resulted in 2 of 5 mice rejecting tumors (Figure 6A). In contrast, all mice from the PancVAX-anti-PD-1 group succumbed to rechallenge (Figure 6, A and $\mathrm{B}$ ).

Finally, to assess whether mice undergoing triple therapy had maintained vaccine-induced neoepitope-specific IFN- $\gamma$-producing $\mathrm{CD}^{+} \mathrm{T}$ cells, splenocytes were harvested at day 22 after rechallenge, stimulated overnight with neoepitopes, and analyzed by ELISPOT (Figure 6C). Mouse 3, 4, and 5, all of which rejected the new tumors and showed complete tumor clearance and displayed robust $\mathrm{T}$ cell responses to at least one neoepitope (Figure 6C). In contrast, mouse 1 failed to reject the new tumor (Figure 6B) and showed limited IFN- $\gamma$-producing $\mathrm{CD}^{+} \mathrm{T}$ cells in response to almost all vaccine peptides (Figure $6 \mathrm{C}$ ). TILs harvested from mouse 1 also showed the absence of IFN- $\gamma$ and TNF- $\alpha$ production as well as a marked number of FoxP $3^{+} \mathrm{CD}^{+} \mathrm{T}$ cells (Figure $6 \mathrm{D}$ ). Taken together, these data demonstrate a vaccine-specific and durable antitumor immune response with the triple therapy.

\section{Discussion}

Neoantigen-targeted vaccines have been shown to induce $\mathrm{T}$ cell responses in immunogenic tumors, such as melanomas, which respond well to checkpoint inhibition. However, this is the first study to our knowledge to demonstrate the induction of neoepitope-specific $\mathrm{T}$ cells with a neoepitope-targeted vaccine in a pancreatic cancer model. Importantly, we show that a neoantigen-targeted vaccine in combination with 
A

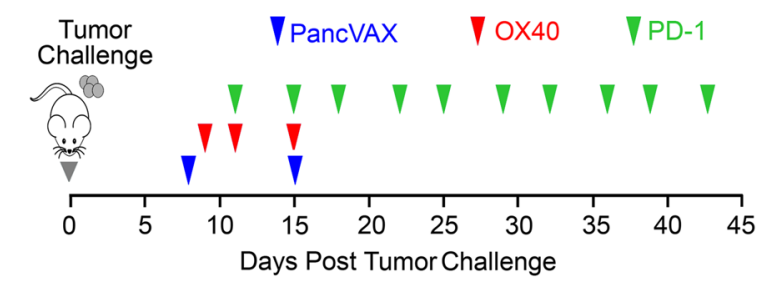

B

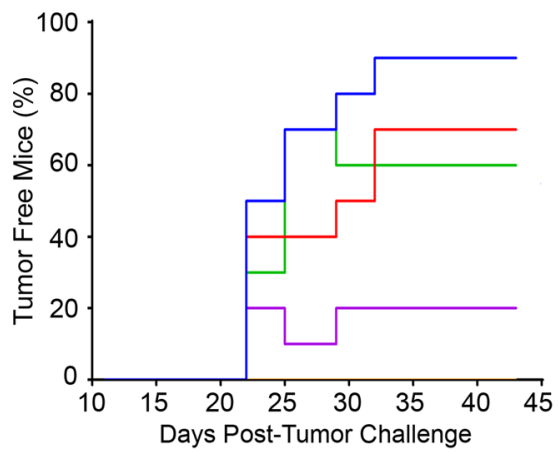

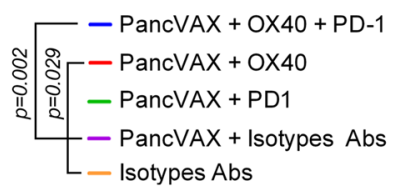

\section{D$$
\text { PancVAX + OX40 + PD-1 }
$$
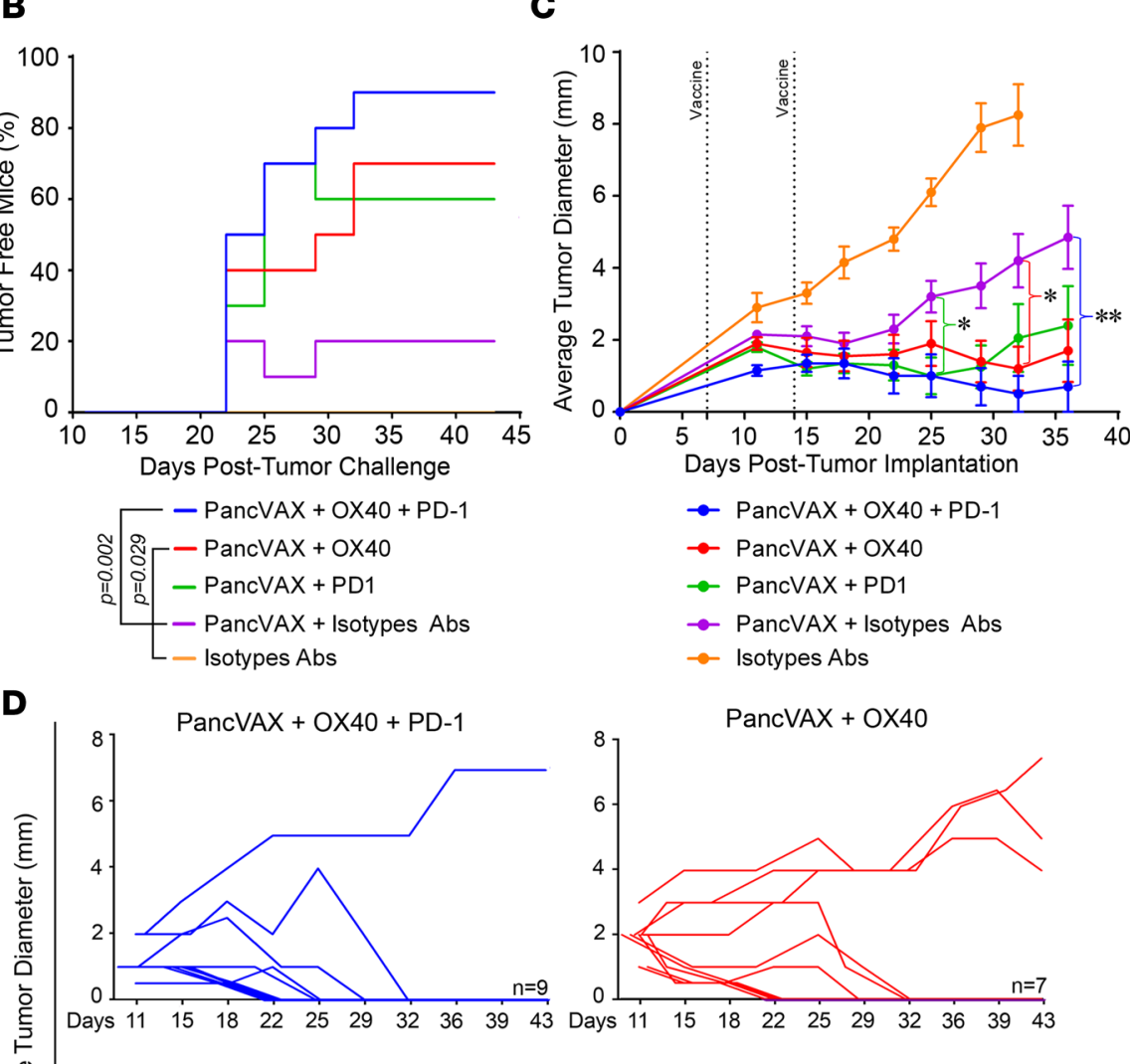

$$
\begin{aligned}
& \rightarrow \text { PancVAX + OX40 + PD-1 } \\
& \rightarrow \text { PancVAX + OX40 } \\
& \rightarrow \text { PancVAX + PD1 } \\
& \rightarrow \text { PancVAX + Isotypes Abs } \\
& \rightarrow \text { Isotypes Abs }
\end{aligned}
$$

PancVAX + OX40

$$
\text { PancVAX + PD-1 }
$$

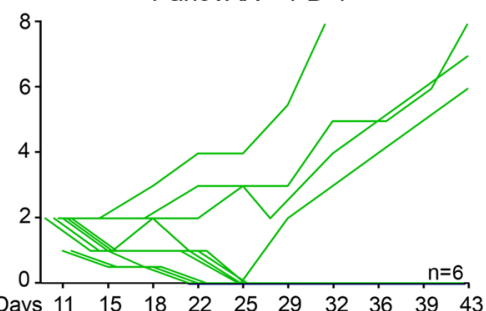

$n=6$

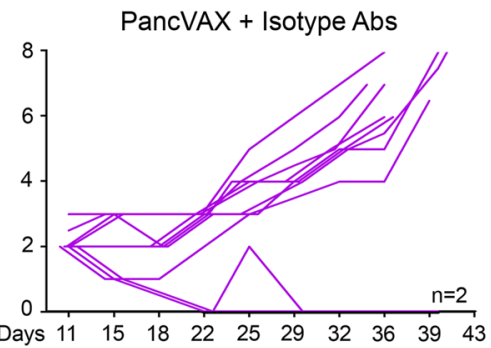

E

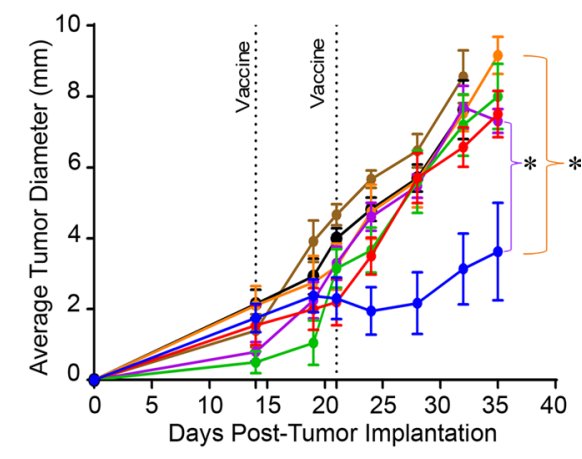

$\rightarrow$ PancVAX + OX40 + PD-1

$\rightarrow$ PancVAX + OX40

$\rightarrow$ PancVAX + PD1

$\rightarrow$ PancVAX + Isotypes Abs

$\rightarrow$ PD-1 + OX40

$\rightarrow$ Isotype Abs

$\mathbf{F}$

$\rightarrow$ Untreated
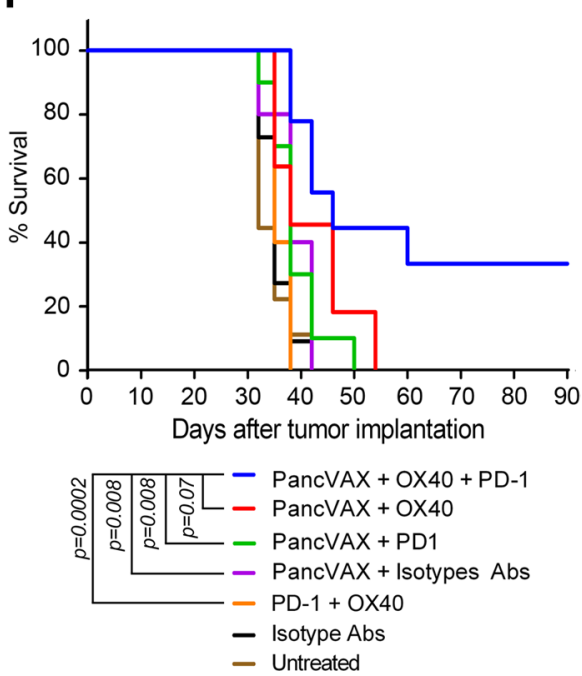

Figure 4. Checkpoint modulation with PancVAX results in tumor clearance. (A) Treatment timeline for Panc02-bearing C57BL6 mice. On the left, 1 week following Panc02 implantation, mice received PancVAX (50 $\mu \mathrm{g}$ each of the 12 20-mer peptides), ADU-V16, AddaVax, agonist OX40 antibody (150 $\mu \mathrm{g})$, and anti-PD-1 antibody (100 $\mu \mathrm{g}$ ), as shown (results in B-D). On the right, PancVAX, ADU-V16, AddaVax, low-dose agonist OX40 antibody (50 $\mu \mathrm{g})$, and anti-PD-1 antibody $(100 \mu \mathrm{g})$ were given 2 weeks after transplantation, as shown (results in $\mathbf{E}$ and $\mathbf{F})$. (B) Kaplan-Meier curves displaying the percentage of tumor-free mice following various treatments (as shown). Statistics by log-rank Mantel-Cox test. (C) Average tumor diameter (mm) was measured in the same group of mice (as in B) by calipers every 3 to 4 days, starting day 11 , until tumors reached $10 \times 10 \mathrm{~mm}$. Statistical comparisons were made with PancVAX plus isotype Abs. ${ }^{*} P<0.05$ for triple therapy from day 25 onward, ${ }^{* *} P<0.002$ at day 35 , triple therapy versus PancVax + Isotype Abs; other groups, namely, PancVAX plus OX40, PancVAX plus PD-1, were nonsignificant, except as noted. Statistics by Student's $t$ test, corrected for multiple comparisons. (D) Average tumor diameter for each mouse for the treatments, as noted. The number indicates the number of mice that were tumor free at day 43. (E) Average tumor diameter $(\mathrm{mm})$ was measured by calipers every 3 to 4 days until tumors reached $10 \times 10$ mm; Statistics by Student's $t$ test adjusted for multiple comparisons. ${ }^{*} P<0.05$, comparing PancVAX, OX 40, and PD- 1 versus $0 X 40$ and PD- 1 or PancVAX and isotype Abs from day 24 onward (except day $28, P=0.082$ for the latter comparison). In all cases 10 mice per group were used at the outset. Of note, mice in whom tumor diameter could not be reliably measured due to poor demarcation of the tumor edge were excluded. (F) Kaplan-Meier curves displaying the percentage of tumor-free mice following various treatments. Statistics by log-rank Mantel-Cox test. 
A

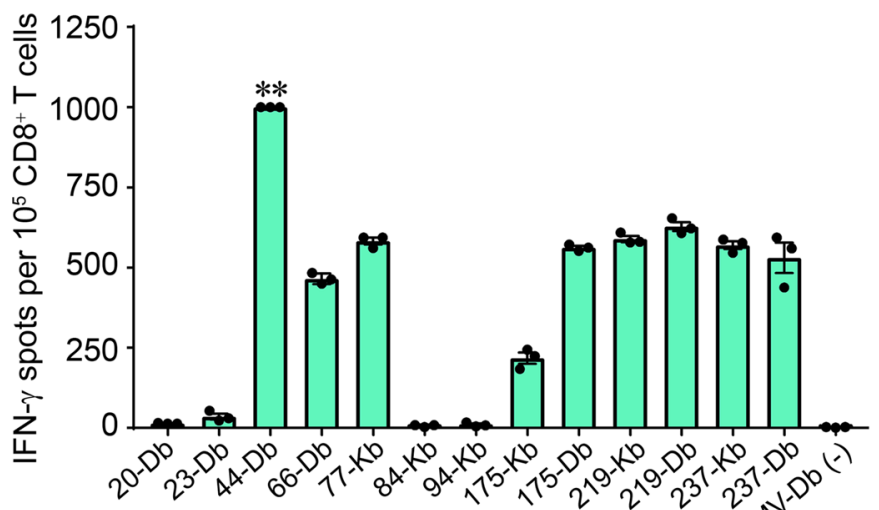

Peptide-MHC allele

C

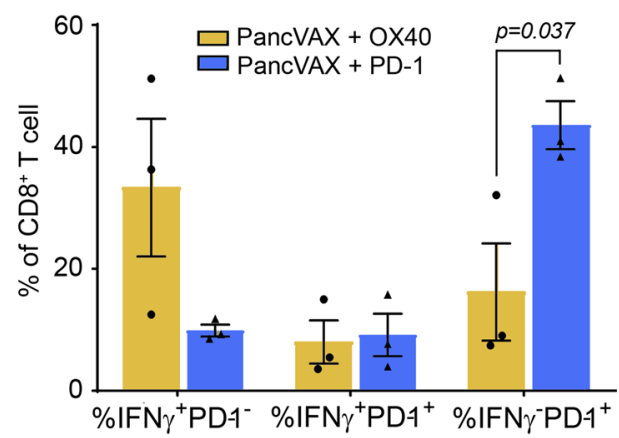

E PancVAX + OX40

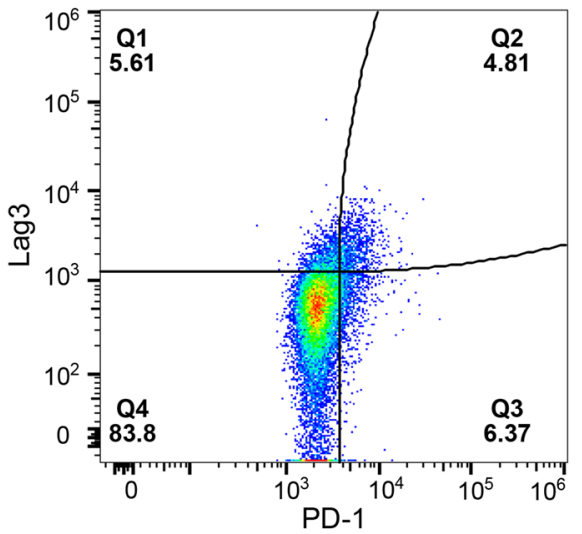

PancVAX + PD-1

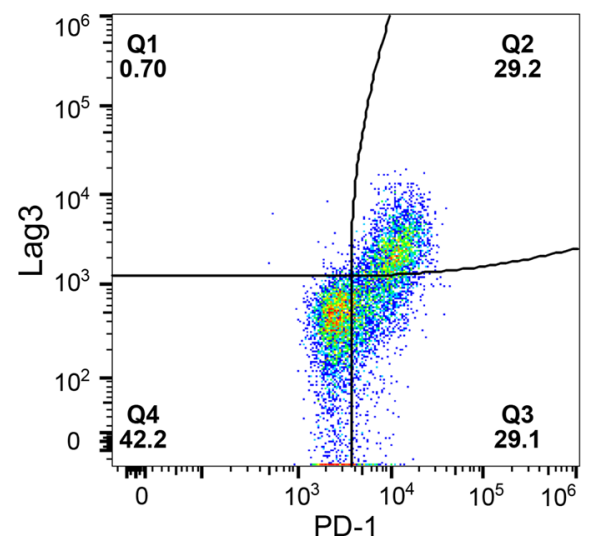

B

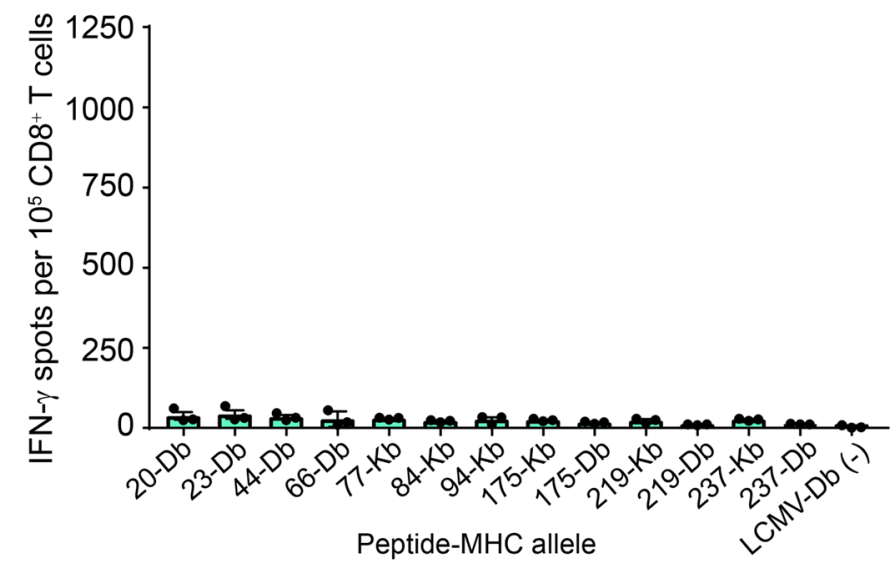

Peptide-MHC allele

$\mathbf{F}$
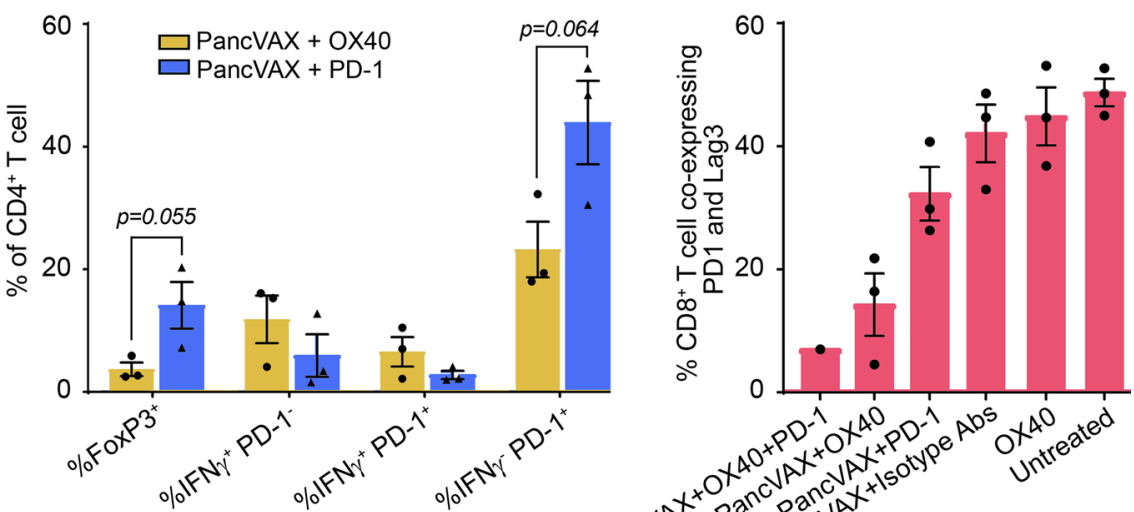

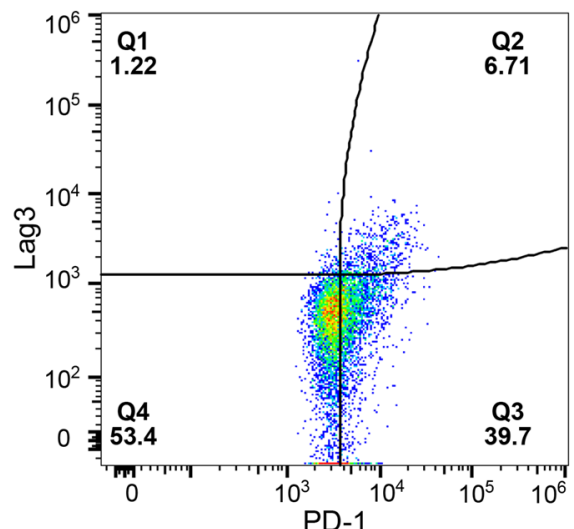

$\mathrm{PD}-1$

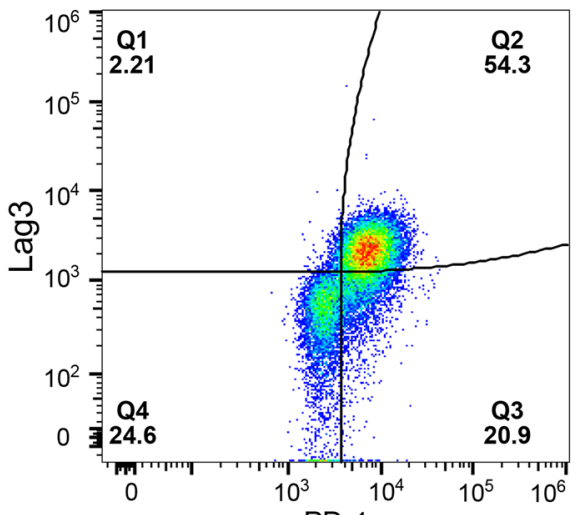

PD-1

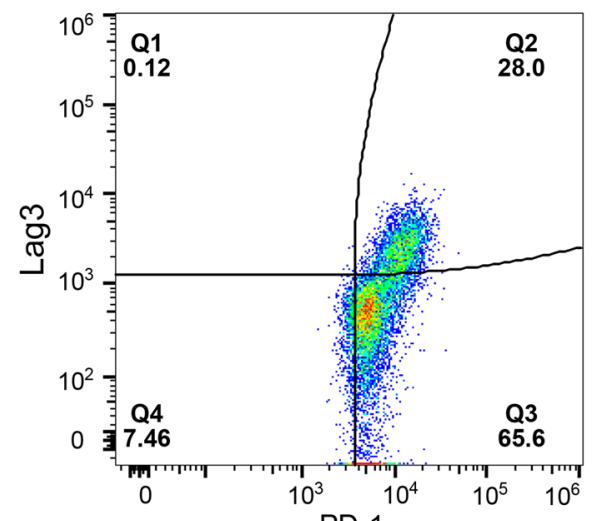

PD-1 
Figure 5. Tumor-infiltrating T cells in mice treated with PancVAX and checkpoint modulators. ELISPOT assays showing IFN- $\gamma$-producing CD8 ${ }^{+} \mathrm{T}$ cells from tumors isolated from mice receiving triple therapy (PancVAX + anti-PD-1+ OX40). ${ }^{*}$ indicates too many spots to count (A) or isotype control is shown in (B). Isolated CD8 $8^{+}$cells were incubated with T2- $D^{\mathrm{b}}$ or $T 2-K^{\mathrm{b}}$ antigen-presenting cells (APCs) that were pulsed with individual PancVAX peptides (as shown). Tumors from 10 mice per group were pooled; technical replicates are shown. (C) Flow cytometry showing the percentage of CD8 ${ }^{+} \mathrm{T}$ cells expressing IFN- $\gamma$, IFN- $\gamma$ with PD-1, or PD-1 alone. (D) Flow cytometry showing the percentage of CD4+ T cells expressing FoxP3, IFN- $\gamma$, IFN- $\gamma$ and PD-1, or PD-1 alone. Each bar represents cells isolated from a single tumor for $\mathbf{C}$ and $\mathbf{D}$. Statistics by unpaired Student's $t$ test. (E) Tumor-infiltrating T cells were harvested and stained for the surface expression of the exhaustion markers Lag3 and PD-1 (flow cytometry) (representative traces from single tumor). (F) Flow cytometry showing the percentage of CD8 $8^{+}$T cells coexpressing PD-1 and Lag3 following treatment of mice with PancVAX, ADU-V16, AddaVax, lowdose OX40 (50 $\mu \mathrm{g})$, and/or anti-PD-1 (100 $\mu \mathrm{g})$. Relevant isotype antibodies were used as controls. For $\mathbf{E}$ and $\mathbf{F}$, cells were gated by size for T cells and then gated for live CD8 ${ }^{+} T$ cells. Statistics by Student's $t$ test, corrected for Bonferroni. $P<0.05 ; n=3$ mice per group (except the triple treatment as the other 2 tumors were cleared); individual mice and mean \pm SEM are shown.

anti-PD-1 and an agonist antibody against OX40 as triple therapy activates and induces vaccine-specific T cells that infiltrate these tumors and cause durable tumor regression to yield a survival benefit. Finally, the triple combination was required to induce the most durable survival benefit, which was associated with a reduced exhausted $\mathrm{T}$ cell population.

While we found that $\mathrm{CD} 8^{+} \mathrm{T}$ cells are the main drivers of PancVAX-induced antitumor immunity, $\mathrm{CD}^{+} \mathrm{T}$ cells may also have a functional role. We noted a complete loss of the antitumor response upon depletion of $\mathrm{CD}^{+}$cells and a partial loss upon $\mathrm{CD}^{+} \mathrm{T}$ cell depletion. Although more studies are required, the latter findings suggest that inclusion of peptides that elicit $\mathrm{CD} 4^{+} \mathrm{T}$ cell responses could further improve tumor clearance. In fact, different models have shown varying requirements for $\mathrm{CD}^{+}$and/or $\mathrm{CD} 8^{+} \mathrm{T}$ cells for tumor eradication. Successes in melanoma patients have nonetheless been associated with immunization, with 20 -mer peptides targeting both $\mathrm{CD}^{+}$and $\mathrm{CD} 8^{+} \mathrm{T}$ cells $(5,20)$.

Interestingly, we did not detect preexisting $\mathrm{T}$ cells specific for the 169 neoantigens in untreated tumor-bearing mice. This absence of preexisting $\mathrm{T}$ cells in response to naturally occurring neoepitopes could arise for several reasons: inadequate antigen presentation due to poor expression of the mutated genes or relatively low MHC I-binding affinities; dominant or preexisting neoantigens falling below the binding affinity cutoff $(\leq 1,000 \mathrm{nM})$; our restriction of the immunogenicity screen to IFN- $\gamma$ production; a low frequency of $\mathrm{T}$ cells capable of recognizing mutant neoepitopes within the repertoire; and/or the $\mathrm{T}$ cells being of low avidity or undergoing apoptosis.

We thus adopted two approaches to enhance vaccine-specific $\mathrm{T}$ cell responses and achieve the most effective tumor clearance. One was to use a potent adjuvant to stimulate innate immunity. It has been shown previously that stimulation of the STING pathway by synthetic CDNs, either via intratumoral injection or administration of whole cell vaccines in combination with synthetic CDNs, robustly induces tumor-specific effector T cells $(4,31)$. Combining the STING adjuvant ADU-V16 with vaccine likewise resulted in a vaccine-specific, polyfunctional $\mathrm{CD}^{+} \mathrm{T}$ cell response. This result is consistent with results from a $\mathrm{Neu} / \mathrm{N}$ breast cancer model, in which combining intratumoral CDNs together with agonist OX40 and anti-PD-1 antibodies resulted in enhanced tumor-specific T cell immunity (36).

Second, we optimized $\mathrm{T}$ cell quality by pairing the neoantigen-targeted vaccine with the appropriate checkpoint modulators. Our results with PancVAX showing enhanced PD-1 expression called for its combination with an anti-PD-1 antibody. Furthermore, we found that an agonist OX40 antibody, when given together with PancVAX, decreased Lag3 and PD-1, the coexpression of which denotes $\mathrm{T}$ cell exhaustion $(37,38)$. Agonist OX40 therapy also skewed the $\mathrm{CD}^{+}{ }^{+} \mathrm{T}$ cell compartment away from an immunosuppressive Treg phenotype toward a Th1 effector phenotype. Consistent with this phenotypic shift, we noted a decreased frequency of FoxP $3^{+} \mathrm{CD}^{+} \mathrm{T}$ cells and the presence of tumor-specific IFN- $\gamma$-secreting $\mathrm{CD} 4^{+} \mathrm{T}$ cells in mice treated with PancVAX plus OX40. We also know that OX40 can improve survival of low-avidity tumor-reactive $\mathrm{T}$ cells and is therefore expected to further enhance antitumor immunity (34).

We used murine Panc02 tumor cells, which may not fully recapitulate human PDA, as the number of expressed neoantigens is higher than the average number of approximately 62 neoantigens documented in human PDA, despite tumor-to-tumor variability (39-41). The alternative was to use the transgenic KPC mouse, but we found that pancreatic tumors in this mouse have too few neoantigen targets, even when compared with human PDA, to be used for a personalized vaccine. This is consistent with published data showing that only 6 expressed missense mutations were identified in the KPC line and none of the 6 mutations had a binding affinity $\mathrm{IC}_{50}$ of $<50 \mathrm{nM}$ or even of $<100 \mathrm{nM}$ (42). Therefore, the KPC model does not adequately recapitulate human tumors in terms of neoantigen burden. 
A

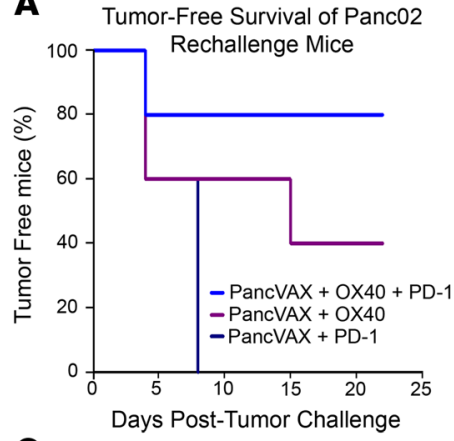

C

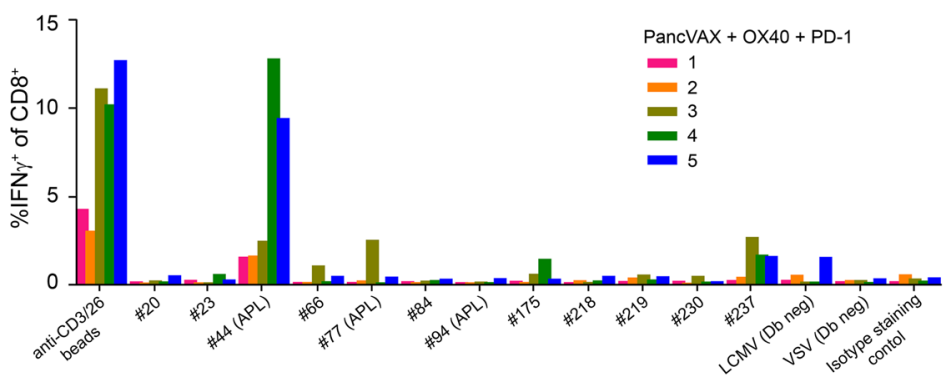

B
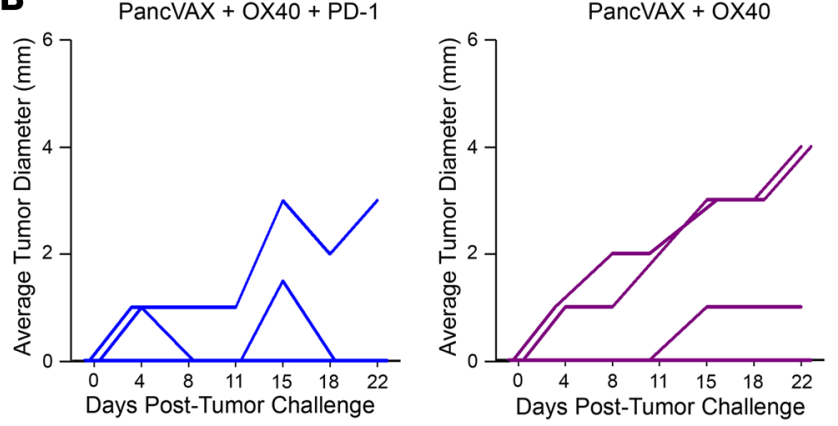

D

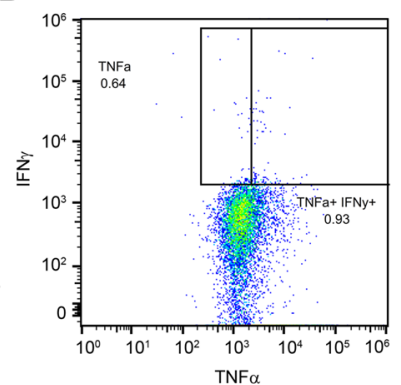

PancVAX + PD-1
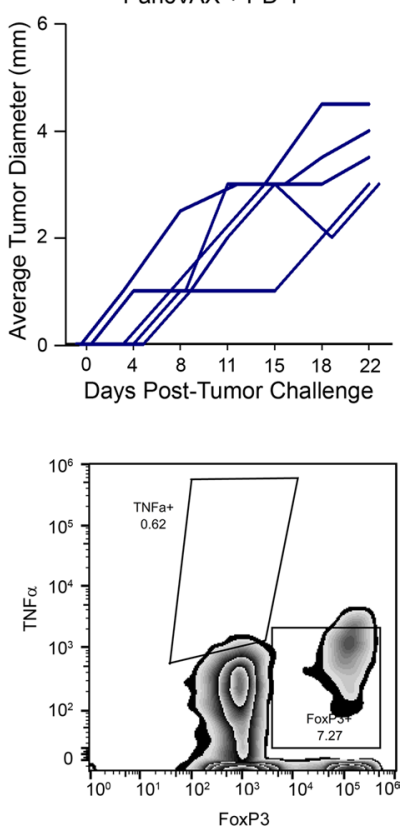

Figure 6. Tumor rechallenge with Panc02 results in sustained protection. (A) $10^{6}$ Panc02 cells were implanted in the contralateral hind legs of mice that had cleared tumor following initial treatments with PancVAX, ADU-V16, AddaVax, OX40, and/or anti-PD-1 (as in Figure 4, B-D) but without additional treatment ( $n=5$ per group). (B) Tumor diameter of individual mice from the groups in $\mathbf{A}$ as a function of time. (C) Splenocytes were harvested from each mouse in the triple treatment group (PancVAX, OX40, and anti-PD-1), plated overnight at $2 \times 10^{6}$ cells in 1 ml CTL medium with each PancVAX peptide $(2 \mu \mathrm{g} / \mathrm{ml})$ or anti-CD3/28 magnetic beads in the presence of protein transport inhibitors. The following day, cells were washed and stained for intracellular cytokine staining to determine peptide specificities. Each number represents a single mouse; mouse 1 did not eradicate the rechallenge tumor. (D) Tumor-infiltrating lymphocytes were harvested from mouse 1, which did not respond to tumor rechallenge following triple therapy and showed the absence of IFN- $\gamma$ and TNF- $\alpha$ production (left) as well as a marked number of immunosuppressive FoxP3+CD4+ Tregs (right).

Furthermore, there is emerging evidence that it is not only the number of neoantigens that is important, rather their "quality" is equally critical $(43,44)$. Based on clinical studies in melanoma, it is clear that only a small minority of epitopes predicted to bind HLA actually elicit an antitumor $\mathrm{CD} 8^{+} \mathrm{T}$ cell response $(5,6)$. Newer studies have demonstrated the significance of a quality metric or "fitness model," which accounts for physical similarity to pathogen-derived antigens, to identify high-quality neoantigens in longterm pancreatic cancer survivors $(43,44)$. In fact, the authors show that the "quality model" was better able to predict overall survival in pancreatic cancer patients compared with the traditional "quantity" model. Therefore, while we know that potentially immunogenic neoantigens exist in pancreatic cancer, we need better paradigms to identify them for vaccine immunotherapy. Further studies will likely explore this aspect of neoantigen selection so as to refine our neoantigen selection pipeline.

In summary, although the Panc02 model does not precisely mirror human PDA in terms of neoantigen burden, it does establish the feasibility of developing a neoantigen-targeted vaccine and, importantly, illustrates the antitumor efficacy of our proposed combinatorial triple therapy in which vaccine is combined with two checkpoint modulators. The model also provides an opportunity to study mechanisms that underpin the inhibition of $\mathrm{T}$ cell activation and infiltration by tumors. Using this model, we provide proof of concept that triple therapy with PancVAX, anti-PD-1, and agonist OX40 elicits a potent antitumor immune response and durable tumor clearance in Panc02-bearing mice by inducing vaccine-specific TILs, lowering the threshold for T cell activation, and reducing TIL exhaustion. These studies illustrate that such therapy may induce and activate vaccine-specific TILs and optimize T cell quality in human PDA, which a single modality alone is unlikely to achieve.

\section{Methods}

Mice and tumor cell lines. The highly tumorigenic murine Panc02 cell line (45) was maintained in DMEM (Life Technologies) supplemented with 10\% fetal bovine serum (Gemini Bio Products), 1\% L-glutamine, and $0.5 \%$ Penicillin/Streptomycin (Life Technologies) in a humidified atmosphere at $37^{\circ} \mathrm{C}$ under $10 \% \mathrm{CO}_{2}$. Male 6-week-old C57BL/6 mice, syngeneic to Panc02, were purchased from 
Jackson Laboratories and allowed to acclimate for 1 week prior to experiments. All mice were housed in pathogen-free conditions.

Identification of immunogenic mutations. NetMHC algorithms (versions 3.2, v3.4, and pan 2.8) $(17,46)$ were used for immunogenicity predictions. Predicted epitopes of lengths of 8-11 amino acids containing the identified nonsynonymous variants were analyzed for affinity to the $\mathrm{H}-2 \mathrm{D}^{\mathrm{b}}$ and $\mathrm{H}-2 \mathrm{~K}^{\mathrm{b}} \mathrm{MHC}$ I molecules. Corresponding 20-mer peptides with mutations centered at position 11 were synthesized by Peptide 2.0 for epitopes, with predictions of $\leq 1,000 \mathrm{nM}$ by any of the 3 prediction algorithms.

Bioinformatics. The full pipeline is available at https://github.com/rosgood/Panc02_Variant_ID. Briefly, the raw sequencing files were aligned to the $\mathrm{mm} 9$ reference genome using bowtie2 (34). Variants were called using freebayes (35) and subsequently annotated using Annovar (36). Tumor-specific peptide sequences were extracted using R (37) and analyzed by a locally maintained NetMHC3.4 software package (38). All mutated epitopes predicted to bind to either $\mathrm{H}-2 \mathrm{~K}^{\mathrm{b}}$ or $\mathrm{H}-2 \mathrm{D}^{\mathrm{b}}$ with an affinity of $\leq 1,000 \mathrm{nM}$ were manually curated by Integrated Genomics Viewer $(39,40)$.

Human comparison of Panc02 cells. We looked for similar human mutations based on the identified Panc02 mutations. For this, genomic coordinates of all mouse mutations used for putative neoantigens were generated from mutect output using the Ensembl database, and the coordinates were queried using the biomaRt $\mathrm{R}$ package. A genomic range including 21 bases surrounding the mutation was then translated to human genomic coordinates using the UCSC liftover tool (https://genome.ucsc.edu/cgi-bin/hgLiftOver). Human mutations occurring within this range were then queried using the ExAC database (http://exac.broadinstitute.org). While no mutations were found at the exact corresponding position for any gene tested, 3 variations were found within the 21-bp range (http://exac.broadinstitute.org/variant/19-572639-C-T, http://exac.broadinstitute.org/variant/2 179401240-C-T, and http://exac.broadinstitute.org/variant/2-179401248-C-T). Only one of these variants was listed in the ClinVar database (www.ncbi.nlm.nih.gov/clinvar), and while it has been associated with various diseases, there is no known cancer association.

Peptides and treatment antibodies. Peptides were synthesized by Peptide 2.0 at $70 \%$ purity for initial screening experiments. After the initial screening, confirmed immunogenic peptides were synthesized at $95 \%$ purity for all further experiments. Lyophilized peptides were stored at $4^{\circ} \mathrm{C}$ with $\mathrm{CaSO}_{4}$ desiccant (Drierite) until needed for experiments, then dissolved in DMSO at $50 \mathrm{mg} / \mathrm{ml}$, aliquoted, and stored at $-80^{\circ} \mathrm{C}$. Once thawed, peptide solutions were kept for no more than 1 month at $4^{\circ} \mathrm{C}$. Therapeutic and depletion antibodies were purchased from BioXCell. For checkpoint blockade studies, $\alpha$-PD-1 (100 $\mu \mathrm{g}$ per injection, clone RMP1-14), its isotype control (rat IgG2a, clone 2A3), and $\alpha$-OX40 (50 or $150 \mu \mathrm{g}$ per injection, clone OX86), or its isotype control (rat IgG1, clone HRPM) were used. All antibodies were injected i.p. in $200 \mu 1$ PBS.

Vaccination protocol for $T$ cell analysis. Groups consisted of 3 mice that each received $10 \mu \mathrm{g}$ of the known $\mathrm{H}-2 \mathrm{~K}^{\mathrm{b}}$-binding ovalbumin (amino acids 152-171) peptide GLEQLESIINFEKLTEWTSS, a pool of 5-6 peptides at $10 \mu \mathrm{g}$ per peptide, and $10 \mu \mathrm{g}$ poly IC:LC as adjuvant (InVivoGen) in PBS. Mice were immunized by injecting a volume of $100 \mu \mathrm{l}$ in the lower hind limb. Seven days later, mice were boosted with the identical vaccine formulation. $\mathrm{CD}^{+} \mathrm{T}$ cell responses were measured 7 days after the boost dose by IFN- $\gamma$ ELISPOT, as described previously (47). 20-mer peptides from LCMV GP L $_{133-52}$ (IITSIKAVYNFATMGILALI), which is known to bind $\mathrm{H}-2 \mathrm{D}^{\mathrm{b}}$, were used as a control for background IFN- $\gamma$ secretion. Variations in the protocol and mouse numbers are described in the Results.

$T$ cell isolation and ELISPOT. CD8 ${ }^{+} \mathrm{T}$ cells were isolated from freshly harvested splenocytes by first creating a single-cell suspension by passing the spleen through a $40-\mu \mathrm{m}$ filter in CTL media (RPMI with $10 \%$ FBS, $0.5 \%$ L-glutamine, $1 \%$ penicillin/streptomycin, and $0.05 \mathrm{mM} 2$-mercaptoethanol). Erythrocytes were removed by ACK lysis, and the resulting T cells were washed, counted, and isolated using the Dynal CD ${ }^{+}$ negative isolation kit (Dynal, Invitrogen) per the manufacturer's instructions. Multiscreen 96-well filtration plates (Millipore) were coated overnight at $4^{\circ} \mathrm{C}$ with $100 \mu 1$ well of anti-mouse IFN- $\gamma$ mAb AN18 (100 $\mu$ g/ $\mathrm{ml}$, Mabtech). Wells were washed 3 times each with PBS and blocked for 2 hours with CTL media at $37^{\circ} \mathrm{C}$. $1 \times 10^{5} \mathrm{~T} 2$ APCs were pulsed with $2 \mu$ g peptide in $100 \mu \mathrm{CTL}$ media for 2 hours at $37^{\circ} \mathrm{C}, 5 \% \mathrm{CO}_{2}$. After T cell isolation, $1 \times 10^{5} \mathrm{CD}^{+} \mathrm{T}$ cells were added to the capture plate in $100 \mu \mathrm{CTL}$, followed by the addition of T2 APCs with peptide and incubation for 18 hours at $37^{\circ} \mathrm{C}$ at $5 \% \mathrm{CO}_{2}$. Cells were removed from the plate by washing 6 times, 2 minutes per wash, with PBS plus $0.05 \%$ Tween20 (MilliporeSigma). Wells were incubated for 2 hours at room temperature with $10 \mu \mathrm{g} / \mathrm{ml}$ biotinylated anti-mouse IFN- $\gamma$ mAb R4-6A2 (Mabtech) in $0.05 \%$ FBS diluted in PBS. Wells were washed as before, incubated with avidin peroxidase complex (Vectastain ELITE ABS kit; Vector Laboratories) for 1 hour at room temperature, and washed 
again. AEC substrate was added, and wells were developed for 10-15 minutes at room temperature. The reaction was stopped with tap water, and plates were allowed to dry for 24 hours before they were counted using an automated image ELISPOT reader (ImmunoSpot).

Murine tumor treatment model. For antitumor experiments, mice received a subcutaneous injection of $1 \times$ $10^{6}$ Panc02 cells in $0.1 \mathrm{ml}$ sterile PBS in the inner flank of the right hind limb on day 0 . Tumor measurements were taken twice weekly beginning on day 10 and average tumor diameter was calculated as length added to width divided by 2 . Mice were sacrificed when tumors reached an average diameter of $10 \mathrm{~mm}$, or began to impede mobility or ulcerate. At this time, spleens and tumors were also harvested for ELISPOT, flow cytometry, or immunofluorescence, as described previously. Peptide vaccine consisting of the 12 expressed immunogenic peptides (Supplemental Table 4; $50 \mu \mathrm{g}$ each) was given on days 3 and 10 together with adjuvant (poly IC:LC or ADU-V16, each with AddaVax) in $100 \mu \mathrm{l}$ of sterile PBS per mouse. Vaccination was performed subcutaneously at the base of the tail. For checkpoint blockade experiments, tumor injections were performed as with T cell depletion studies, but treatment was delayed until tumors were palpable, at day 10.

TIL isolation. Tumors were excised immediately after mice were euthanized and placed into CTL media. They were diced into small pieces, incubated with digestion media (DMEM, 25 mg/1 Hyaluronidase, $1 \mathrm{~g} / 1$ Collagenase IV; all from Life Technologies), incubated at $37^{\circ} \mathrm{C}$ for 30 minutes with shaking, and then centrifuged for 10 minutes at $500 \mathrm{~g}$. Tumor samples were washed once with RPMI (Life Technologies), filtered through a $70-\mu \mathrm{m}$ filter (Falcon), and plated for 1 hour at $37^{\circ} \mathrm{C}, 5 \% \mathrm{CO}_{2}$ to remove adherent tumor cells

Intracellular cytokine staining and flow cytometry. Isolated cells were stimulated overnight (18 hours) with peptide or anti-CD3/28 magnetic beads (Gibco) in the presence of protein transport inhibitor cocktail (eBioscience). Cells were washed with PBS, stained with Live/Dead Fixable Aqua (Life Technologies) for 20 minutes on ice in the dark, washed 3 times with PBS, and stained for surface markers diluted in FACS buffer (PBS with $1 \% \mathrm{FBS}$ and $0.1 \% \mathrm{NaN}_{3}$ ) for 30 minutes on ice in the dark. Cells were washed 3 times with FACS buffer, fixed and permeabilized using the FoxP3 Fix/Perm kit (eBioscience) for 20 minutes at room temperature in the dark, washed once with Perm/Wash buffer (eBioscience), and stained for intracellular markers. After washing 3 times, cells were resuspended in FACS buffer and either run on a Gallios flow cytometer (Beckman Coulter) immediately or after overnight storage at $4^{\circ} \mathrm{C}$ in the dark. Analysis was performed using FlowJo (Treestar).

Statistics. Statistical analysis was performed using GraphPad Prism software. All data are presented as mean \pm SEM. For survival curve analysis, the log-rank Mantel-Cox test was used. ANOVA or 2-tailed Student's $t$ test with Bonferroni's correction was used for multiple comparisons. Differences between groups were considered significant at $P \leq 0.05$.

Study approval. All mice were housed in pathogen-free conditions and treated in accordance with Johns Hopkins University School of Medicine Institutional Animal Care and Use Committee and American Association of Laboratory Animal Committee-approved policies.

\section{Author contributions}

HLK performed bulk of the experiments, analyzed data, and helped write and edit the manuscript. AH, TV, MY, AAW, EL, KC, and SW assisted with experiments and data analysis and edited the manuscript. LHG, CON, SMM, and TWD developed and provided the STING adjuvant, helped optimize the adjuvant proto$\mathrm{col}$, and edited the manuscript. TDA supervised and helped design experiments, analyzed data, and edited the manuscript. EMJ supervised the design and analysis of all experiments and wrote and edited the manuscript. NZ performed experiments, analyzed data, performed statistics, and wrote and edited the manuscript.

\section{Acknowledgments}

The authors gratefully acknowledge the James W. and Frances Gibson McGlothlin Foundation; the Skip Viragh Center for Pancreatic Cancer at Johns Hopkins; The Bloomberg Kimmel Institute for Cancer Immunotherapy at Johns Hopkins; the National Cancer Institute (R01 CA18492-04 and R01 CA19729603 to EMJ); the Pancreatic Cancer Action Network-American Association for Cancer Research Career Development Award (13-20-25-LUTZ to EL); and a Stand Up To Cancer-Lustgarten Foundation Pancreatic Cancer Convergence Dream Team Translational Research Grant (SU2C-AACR-DT1414 to EMJ). AAW is supported by NIH grant T32 GM007309-40. NZ is supported in part by NIH grant T32CA00971-37 and is recipient of the American Society of Clinical Oncology Young Investigator Award. 
Address correspondence to: Elizabeth M. Jaffee or Neeha Zaidi, Room 4M07 Cancer Research Building I, Suite 2-103, 1830 E. Monument St., Baltimore, Maryland 21205, USA. Phone: 410.955 .2957 (E.M. Jaffee and N. Zaidi); Email: ejaffee@jhmi.edu (E.M. Jaffee). Email: nzaidi1@jhmi.edu (N. Zaidi).

1. Le DT, et al. PD-1 Blockade in tumors with mismatch-repair deficiency. N Engl J Med. 2015;372(26):2509-2520.

2. Schumacher TN, Schreiber RD. Neoantigens in cancer immunotherapy. Science. 2015;348(6230):69-74.

3. Yarchoan M, Johnson BA, Lutz ER, Laheru DA, Jaffee EM. Targeting neoantigens to augment antitumour immunity. Nat Rev Cancer. 2017;17(4):209-222.

4. Carreno BM, et al. Cancer immunotherapy. A dendritic cell vaccine increases the breadth and diversity of melanoma neoantigen-specific T cells. Science. 2015;348(6236):803-808.

5. Ott PA, et al. An immunogenic personal neoantigen vaccine for patients with melanoma. Nature. 2017;547(7662):217-221

6. Sahin U, et al. Personalized RNA mutanome vaccines mobilize poly-specific therapeutic immunity against cancer. Nature. 2017;547(7662):222-226.

7. Rizvi NA, et al. Cancer immunology. Mutational landscape determines sensitivity to PD-1 blockade in non-small cell lung cancer Science. 2015;348(6230):124-128.

8. Snyder A, et al. Genetic basis for clinical response to CTLA-4 blockade in melanoma. N Engl J Med. 2014;371(23):2189-2199.

9. Yarchoan M, Hopkins A, Jaffee EM. Tumor mutational burden and response rate to PD-1 inhibition. N Engl J Med. 2017;377(25):2500-2501.

10. Vogelstein B, Papadopoulos N, Velculescu VE, Zhou S, Diaz LA, Kinzler KW. Cancer genome landscapes. Science. 2013;339(6127):1546-1558.

11. Royal RE, et al. Phase 2 trial of single agent Ipilimumab (anti-CTLA-4) for locally advanced or metastatic pancreatic adenocarcinoma. J Immunother. 2010;33(8):828-833.

12. Lutz ER, et al. Immunotherapy converts nonimmunogenic pancreatic tumors into immunogenic foci of immune regulation. Cancer Immunol Res. 2014;2(7):616-631.

13. Le DT, et al. Evaluation of ipilimumab in combination with allogeneic pancreatic tumor cells transfected with a GM-CSF gene in previously treated pancreatic cancer. J Immunother. 2013;36(7):382-389.

14. Mouse Genome Sequencing Consortium, et al. Initial sequencing and comparative analysis of the mouse genome. Nature. 2002;420(6915):520-562.

15. Robinson JT, et al. Integrative genomics viewer. Nat Biotechnol. 2011;29(1):24-26.

16. Thorvaldsdóttir H, Robinson JT, Mesirov JP. Integrative Genomics Viewer (IGV): high-performance genomics data visualization and exploration. Brief Bioinformatics. 2013;14(2):178-192.

17. Lundegaard C, Lund O, Nielsen M. Accurate approximation method for prediction of class I MHC affinities for peptides of length 8, 10 and 11 using prediction tools trained on 9mers. Bioinformatics. 2008;24(11):1397-1398.

18. Slansky JE, et al. Enhanced antigen-specific antitumor immunity with altered peptide ligands that stabilize the MHC-peptideTCR complex. Immunity. 2000;13(4):529-538.

19. Salter RD, Cresswell P. Impaired assembly and transport of HLA-A and -B antigens in a mutant TxB cell hybrid. EMBO J. 1986;5(5):943-949.

20. Gubin MM, et al. Checkpoint blockade cancer immunotherapy targets tumour-specific mutant antigens. Nature. 2014;515(7528):577-581.

21. Leao IC, Ganesan P, Armstrong TD, Jaffee EM. Effective depletion of regulatory T cells allows the recruitment of mesothelin-specific CD8 T cells to the antitumor immune response against a mesothelin-expressing mouse pancreatic adenocarcinoma. Clin Transl Sci. 2008;1(3):228-239.

22. Celis E. Toll-like receptor ligands energize peptide vaccines through multiple paths. Cancer Res. 2007;67(17):7945-7947.

23. Currie AJ, van der Most RG, Broomfield SA, Prosser AC, Tovey MG, Robinson BW. Targeting the effector site with IFN-alphabeta-inducing TLR ligands reactivates tumor-resident CD8 T cell responses to eradicate established solid tumors. J Immunol. 2008;180(3):1535-1544.

24. Pulko V, et al. TLR3-stimulated dendritic cells up-regulate B7-H1 expression and influence the magnitude of CD8 T cell responses to tumor vaccination. J Immunol. 2009;183(6):3634-3641.

25. Salem ML, Kadima AN, Cole DJ, Gillanders WE. Defining the antigen-specific T-cell response to vaccination and poly(I:C)/ TLR3 signaling: evidence of enhanced primary and memory CD8 T-cell responses and antitumor immunity. J Immunother. 2005;28(3):220-228.

26. Corrales L, et al. Direct activation of STING in the tumor microenvironment leads to potent and systemic tumor regression and immunity. Cell Rep. 2015;11(7):1018-1030.

27. Aucouturier J, Dupuis L, Deville S, Ascarateil S, Ganne V. Montanide ISA 720 and 51: a new generation of water in oil emulsions as adjuvants for human vaccines. Expert Rev Vaccines. 2002;1(1):111-118.

28. Aucouturier J, Dupuis L, Ganne V. Adjuvants designed for veterinary and human vaccines. Vaccine. 2001;19(17-19):2666-2672.

29. Calabro S, et al. The adjuvant effect of MF59 is due to the oil-in-water emulsion formulation, none of the individual components induce a comparable adjuvant effect. Vaccine. 2013;31(33):3363-3369.

30. Ott G, Barchfeld GL, Chernoff D, Radhakrishnan R, van Hoogevest P, Van Nest G. MF59. Design and evaluation of a safe and potent adjuvant for human vaccines. Pharm Biotechnol. 1995;6:277-296.

31. Dubensky TW, Kanne DB, Leong ML. Rationale, progress and development of vaccines utilizing STING-activating cyclic dinucleotide adjuvants. Ther Adv Vaccines. 2013;1(4):131-143.

32. Wherry EJ, Kurachi M. Molecular and cellular insights into T cell exhaustion. Nat Rev Immunol. 2015;15(8):486-499.

33. Soares KC, et al. PD-1/PD-L1 blockade together with vaccine therapy facilitates effector T-cell infiltration into pancreatic tumors. J Immunother. 2015;38(1):1-11. 
34. Black CM, Armstrong TD, Jaffee EM. Apoptosis-regulated low-avidity cancer-specific CD8(+) T cells can be rescued to eliminate HER2/neu-expressing tumors by costimulatory agonists in tolerized mice. Cancer Immunol Res. 2014;2(4):307-319.

35. Murata S, et al. OX40 costimulation synergizes with GM-CSF whole-cell vaccination to overcome established CD8+ T cell tolerance to an endogenous tumor antigen. J Immunol. 2006;176(2):974-983.

36. Foote JB, et al. A STING agonist given with OX40 receptor and PD-L1 modulators primes immunity and reduces tumor growth in tolerized mice. Cancer Immunol Res. 2017;5(6):468-479.

37. Huang RY, Eppolito C, Lele S, Shrikant P, Matsuzaki J, Odunsi K. LAG3 and PD1 co-inhibitory molecules collaborate to limit CD8+ T cell signaling and dampen antitumor immunity in a murine ovarian cancer model. Oncotarget. 2015;6(29):27359-27377.

38. Woo SR, et al. Immune inhibitory molecules LAG-3 and PD-1 synergistically regulate T-cell function to promote tumoral immune escape. Cancer Res. 2012;72(4):917-927.

39. Bailey P, et al. Exploiting the neoantigen landscape for immunotherapy of pancreatic ductal adenocarcinoma. Sci Rep 2016;6:35848

40. Bailey P, et al. Genomic analyses identify molecular subtypes of pancreatic cancer. Nature. 2016;531(7592):47-52

41. Knudsen ES, et al. Stratification of pancreatic ductal adenocarcinoma: combinatorial genetic, stromal, and immunologic markers. Clin Cancer Res. 2017;23(15):4429-4440.

42. Evans RA, et al. Lack of immunoediting in murine pancreatic cancer reversed with neoantigen. JCI Insight. 2016;1(14):e88328

43. Balachandran VP, et al. Identification of unique neoantigen qualities in long-term survivors of pancreatic cancer. Nature. 2017;551(7681):512-516.

44. Euksza M, et al. A neoantigen fitness model predicts tumour response to checkpoint blockade immunotherapy. Nature. 2017;551(7681):517-520.

45. Corbett $\mathrm{TH}$, et al. Induction and chemotherapeutic response of two transplantable ductal adenocarcinomas of the pancreas in C57BL/6 mice. Cancer Res. 1984;44(2):717-726.

46. Hoof I, et al. NetMHCpan, a method for MHC class I binding prediction beyond humans. Immunogenetics. 2009;61(1):1-13

47. Thomas AM, et al. Mesothelin-specific CD8(+) T cell responses provide evidence of in vivo cross-priming by antigen-presenting cells in vaccinated pancreatic cancer patients. J Exp Med. 2004;200(3):297-306 